\title{
Resolvent and logarithmic residues of a singular operator pencil in Hilbert spaces
}

\author{
Massimo Franchi ${ }^{1}$ \\ Department of Statistical Sciences, Sapienza University of Rome, P.le Aldo Moro \\ 5, 00185 Rome, Italy
}

\section{A R T I C L E I N F O}

Article history:

Received 21 May 2020

Accepted 13 January 2022

Available online 14 January 2022

Submitted by H. Bart

\section{$M S C:$}

$15 \mathrm{~A} 18$

$15 \mathrm{~A} 21$

$15 \mathrm{~A} 23$

$47 \mathrm{~A} 10$

$47 \mathrm{~A} 11$

$47 \mathrm{~A} 15$

\section{Keywords:}

Local spectral properties

Chains

Resolvent

Factorizations

Logarithmic residues

\section{A B S T R A C T}

The present paper considers the operator pencil $A(\lambda)=$ $A_{0}+A_{1} \lambda$, where $A_{0}, A_{1} \neq 0$ are bounded linear mappings between complex Hilbert spaces and $A_{0}$ is neither one-toone nor onto. Assuming that 0 is an isolated singularity of $A(\lambda)$ and that the image of $A_{0}$ is closed, certain operators are defined recursively starting from $A_{0}$ and $A_{1}$ and they are shown to provide a characterization of the image and null space of the operators in the principal part of the resolvent and of the logarithmic residues of $A(\lambda)$ at 0 . The relations with the classical results based on ascent and descent in [10] are discussed. In the special case of $A_{0}$ being Fredholm of index 0 , the present results characterize the rank of the operators in the principal part of the resolvent, the dimension of the subspaces that define the ascent and descent, the partial multiplicities, and the algebraic multiplicity of $A(\lambda)$ at 0 .

(C) 2022 Elsevier Inc. All rights reserved.

\footnotetext{
E-mail address: massimo.franchi@uniroma1.it.

1 I would like to thank an anonymous referee and the handling editor (Harm Bart) for many valuable comments on previous versions of the paper. Partial financial support from Sapienza University of Rome Ateneo 2018 grant is gratefully acknowledged.
} 


\section{Introduction}

Let $\mathscr{B}_{X, Y}$ be the space of bounded linear mappings between two complex Hilbert spaces $X$ and $Y$ (when $X=Y$, the shorthand notation $\mathscr{B}_{X}=\mathscr{B}_{X, X}$ is employed) and consider the operator pencil ${ }^{2}$

$$
A(\lambda)=A_{0}+A_{1} \lambda, \quad A_{0}, A_{1} \in \mathscr{B}_{X, Y}, \quad A_{0}, A_{1} \neq 0, \quad \lambda \in \Omega \subseteq \mathbb{C} .
$$

Assume that $A_{0}$ is neither one-to-one nor onto and that there exists $\rho>0$ such that $A(\lambda)$ is invertible for all $\lambda \in D_{\rho} \backslash\{0\}$, where $D_{\rho}=\{\lambda \in \mathbb{C}:|\lambda|<\rho\}$. That is, 0 is an isolated singularity of $A(\lambda) .^{3}$

Under the assumption that the image of $A_{0}$ is closed and that the resolvent $A(\lambda)^{-1}$ has a pole of some order $m \in \mathbb{N}$ at 0 , the present paper provides a construction of the resolvent and of the logarithmic residues and a characterization of the image and null space of those operators. The quantities are all expressed in terms of the image and null space of certain operators defined recursively by a procedure called 'local subspace decomposition', see Definition 2.2 below, which only requires knowledge of $A_{0}, A_{1}$.

This recursive procedure was introduced in $[12,13]$ for the finite dimensional case $X=Y=\mathbb{C}^{p}$ and it was called 'local rank factorization'. Franchi and Paruolo [13] also showed that the (extended) local rank factorization coincides with the 'complete reduction process' in [6]; the latter builds on the results of [21] and on the reduction technique in [20], [19] and delivers the order of the pole and the coefficients of the resolvent. The book by [5] contains a thorough treatment of their approach and its many extensions, see e.g. [22] and [1-4] for the infinite dimensional Hilbert and Banach space cases. The local rank factorization was further employed in [14] and [11] for eigenvalues of finite type in the (possibly infinite dimensional) Hilbert space case $X=Y$.

In the setup considered in the present paper, the notion of rank may not be well defined and thus the name 'local rank factorization' is misleading. Because the procedure depends only on the sequence of subspaces determined by the image and null space of certain operators (and not on their dimensions), the name 'local subspace decomposition' is used here instead.

From the theory of essential singularities in Banach spaces in [2], it is well known that the resolvent of (1.1) admits the Laurent series representation

$$
A(\lambda)^{-1}=\sum_{n \in \mathbb{Z}} R_{n} \lambda^{n}, \quad \lambda \in U_{\sigma, \rho}=\{\lambda \in \mathbb{C}: \sigma<|\lambda|<\rho\}, \quad 0 \leq \sigma<\rho \leq \infty,
$$

if and only if the operators $R_{n} \in \mathscr{B}_{Y, X}$ are suitably bounded, i.e.

\footnotetext{
${ }^{2}$ In the following, 0 and $I$ respectively denote the zero and the identity in the different ambient spaces (the relevant space should be clear from the context).

3 By the change of variable $\left(\lambda-\lambda_{0}\right) \mapsto \lambda$, this setup handles isolated singularities at points $\lambda_{0}$ other than zero. In fact, if $\lambda_{0} \neq 0$ is such that $A\left(\lambda_{0}\right)=A_{0}+\lambda_{0} A_{1}$ is not invertible then $A(\lambda)=A_{0}+\lambda_{0} A_{1}+$ $A_{1} \lambda-\lambda_{0} A_{1}=\widetilde{A}_{0}+A_{1}\left(\lambda-\lambda_{0}\right)$, where $\widetilde{A}_{0}=A_{0}+\lambda_{0} A_{1}$ is singular. Hence there is no loss of generality in assuming that $\lambda_{0}=0$ is the point at which $A(\lambda)$ is singular.
} 


$$
\lim _{n \rightarrow \infty}\left\|R_{-n}\right\|^{1 / n} \leq \sigma, \quad \lim _{n \rightarrow \infty}\left\|R_{n}\right\|^{1 / n} \leq 1 / \rho
$$

and satisfy the 'fundamental equations'

$$
A_{0} R_{n}+A_{1} R_{n-1}=1_{n=0} I, \quad R_{n} A_{0}+R_{n-1} A_{1}=1_{n=0} I, \quad n \in \mathbb{Z},
$$

where $1_{n=h}$ is the indicator function (equal to 1 if $n=h$ and to 0 otherwise); these follow from the identities $A(\lambda) A(\lambda)^{-1}=I \in \mathscr{B}_{Y}$ and $A(\lambda)^{-1} A(\lambda)=I \in \mathscr{B}_{X}$.

Albrecht et al. [2] show that (1.3) and (1.4) hold if and only if (i) $P=R_{-1} A_{1}$ and $P^{c}=I-P=R_{0} A_{0}$ are complementary projections on $X$ and $Q=A_{1} R_{-1}$ and $Q^{c}=I-Q=A_{0} R_{0}$ are complementary projections on $Y,(i i) A_{n}=Q A_{n} P+Q^{c} A_{n} P^{c}$, $n=0,1,($ iii $) R_{n}=P R_{n} Q, n=-1,-2, \ldots$, and $R_{n}=P^{c} R_{n} Q^{c}, n=0,1, \ldots$,

$$
R_{n}= \begin{cases}(-1)^{-n-1}\left(R_{-1} A_{0}\right)^{-n-1} R_{-1}, & n=-1,-2, \ldots \\ (-1)^{n}\left(R_{0} A_{1}\right)^{n} R_{0}, & n=0,1, \ldots\end{cases}
$$

and $(v) \lim _{n \rightarrow \infty}\left\|\left(R_{-1} A_{0}\right)^{n}\right\|^{1 / n} \leq \sigma, \lim _{n \rightarrow \infty}\left\|\left(R_{0} A_{1}\right)^{n}\right\|^{1 / n} \leq 1 / \rho$. The formulas in (iv) extend those derived for the matrix case in [25,26], see also [28]. Note that $A(\lambda)$ (and thus $A(\lambda)^{-1}$ ) is completely reduced by the complementary projections in $(i)$ and further observe that, when $R_{-1} A_{0}$ is nilpotent, the theory covers poles as a particular case.

However, as observed in Remark 1 in [4], $R_{n}, n \in \mathbb{Z}$, is unknown; hence these formulas cannot be used per se to calculate the complementary projections and the coefficients of the resolvent because they are all expressed in terms of $R_{0}, R_{-1}$. Albrecht et al. [4] define infinite-length singular and regular Jordan chains that determine the complementary projections $P, P^{c}, Q, Q^{c}$, and, when $X, Y$ are separable, they find $R_{0}, R_{-1}$ by solving recursively the projected versions of the fundamental equations. They then employ (iv) to calculate the resolvent.

The present paper considers the special case in which $X$ and $Y$ are Hilbert spaces and the resolvent has a pole of some order $m \in \mathbb{N}$ at 0 , i.e. $R_{-m} \neq 0$ and $R_{-m-n}=0$ for $n=1,2, \ldots$ As shown in [2], in this case the annulus of convergence of (1.2) becomes the punctured $\operatorname{disc} U_{0, \rho}=\{\lambda \in \mathbb{C}: 0<|\lambda|<\rho\}=D_{\rho} \backslash\{0\}$; here we set $Z_{n}=R_{-m+n}$ for $n=0,1, \ldots$ and write $(1.2)$ as

$$
A(\lambda)^{-1}=\sum_{n=0}^{\infty} Z_{n} \lambda^{n-m}, \quad \lambda \in D_{\rho} \backslash\{0\}, \quad Z_{0} \neq 0 .
$$

From the classical theory of poles in Banach spaces in Bart and Lay [10], see also [16], [24], [29], [23], [27], it is well known that the resolvent has a pole of order $m$ at 0 if and only if the ascent and the descent (defined therein in terms of certain subspaces $\left.\mathscr{N}_{n}, \mathscr{R}_{n}, \mathscr{N}_{n}^{\prime}, \mathscr{R}_{n}^{\prime}, n=0,1, \ldots\right)$ equal $m$. In this case, the direct sum decompositions

$$
X=\mathscr{N}_{m} \oplus \mathscr{R}_{m}, \quad Y=\mathscr{N}_{m}^{\prime} \oplus \mathscr{R}_{m}^{\prime}
$$


where $\mathscr{N}_{m}, \mathscr{R}_{m}, \mathscr{N}_{m}^{\prime}$, and $\mathscr{R}_{m}^{\prime}$ are closed subspaces, completely reduce $A(\lambda)$ (and thus $\left.A(\lambda)^{-1}\right)$; that is, relative to them, $A(\lambda)$ admits the operator matrix representation

$$
A(\lambda)=\left(\begin{array}{cc}
A_{0 \mathscr{N}}+A_{1 \mathscr{N}} \lambda & 0 \\
0 & A_{0 \mathscr{R}}+A_{1 \mathscr{R}} \lambda
\end{array}\right), \quad A_{n \mathscr{Z}}=A_{n} \mid \mathscr{Z}_{m}: \mathscr{Z}_{m} \mapsto \mathscr{Z}_{m}^{\prime},
$$

where $A_{n \mathscr{N}}$ denotes the restriction of $A_{n}$ to $\mathscr{N}_{m}$ and maps $\mathscr{N}_{m}$ to $\mathscr{N}_{m}^{\prime}$ and $A_{n \mathscr{R}}$ is the restriction of $A_{n}$ to $\mathscr{R}_{m}$ mapping $\mathscr{R}_{m}$ to $\mathscr{R}_{m}^{\prime}$; thus

$$
A(\lambda)^{-1}=\left(\begin{array}{cc}
\left(A_{0 \mathscr{N}}+A_{1 \mathscr{N}} \lambda\right)^{-1} & 0 \\
0 & \left(A_{0 \mathscr{R}}+A_{1 \mathscr{R}} \lambda\right)^{-1}
\end{array}\right)
$$

Because $A_{1 \mathscr{N}}$ and $A_{0 \mathscr{R}}$ are invertible and $N=\left(A_{1 \mathscr{N}}\right)^{-1} A_{0 \mathscr{N}}$ is nilpotent with index $m$, i.e. $N^{m}=0$ and $N^{m-1} \neq 0$, the principal part is

$$
\left(A_{0 \mathscr{N}}+A_{1 \mathscr{N}} \lambda\right)^{-1}=\sum_{n=0}^{m-1} Z_{n \mathscr{N}^{\prime}} \lambda^{n-m}, \quad Z_{n \mathscr{N}^{\prime}}=(-1)^{m-1-n} N^{m-1-n} A_{1 \mathscr{N}}^{-1}
$$

where $Z_{n \mathscr{N}^{\prime}}$ is the restriction of $Z_{n}$ to $\mathscr{N}_{m}^{\prime}$ mapping $\mathscr{N}_{m}^{\prime}$ to $\mathscr{N}_{m}$, and the regular part is

$$
\begin{gathered}
\left(A_{0 \mathscr{R}}+A_{1 \mathscr{R}} \lambda\right)^{-1}=\sum_{n=m}^{\infty} Z_{n \mathscr{R}^{\prime}} \lambda^{n-m}, \\
Z_{n \mathscr{R}^{\prime}}=\left\{\begin{array}{cc}
A_{0 \mathscr{R}}^{-1} & n=m \\
-A_{0 \mathscr{R}}^{-1} A_{1 \mathscr{R}} Z_{n-1 \mathscr{R}^{\prime}} & n=m+1, m+2, \ldots
\end{array},\right.
\end{gathered}
$$

where $Z_{n \mathscr{R}^{\prime}}$ is the restriction of $Z_{n}$ to $\mathscr{R}_{m}^{\prime}$ mapping $\mathscr{R}_{m}^{\prime}$ to $\mathscr{R}_{m}$. This shows that, relative to the direct sum decompositions in (1.6), the resolvent admits the operator matrix representation

$$
A(\lambda)^{-1}=\sum_{n=m}^{\infty}\left(\begin{array}{cc}
Z_{n \mathscr{N}^{\prime}} & 0 \\
0 & 0
\end{array}\right) \lambda^{n-m}+\sum_{n=m}^{\infty}\left(\begin{array}{cc}
0 & 0 \\
0 & Z_{n \mathscr{R}^{\prime}}
\end{array}\right) \lambda^{n-m}
$$

from which one can construct $Z_{n}$ in (1.5) by a change of bases. Finally recall that, letting $P_{\mathscr{N}, \mathscr{R}}, P_{\mathscr{R}, \mathscr{N}}=I-P_{\mathscr{N}, \mathscr{R}}$ and $P_{\mathscr{N}^{\prime}, \mathscr{R}^{\prime}}, P_{\mathscr{R}^{\prime}, \mathscr{N}^{\prime}}=I-P_{\mathscr{N}^{\prime}, \mathscr{R}^{\prime}}$ be the projections associated to the direct sum decompositions in (1.6), one has

$$
\begin{aligned}
P_{\mathscr{N}, \mathscr{R}} & =\frac{1}{2 \pi i} \int_{\partial D_{\rho}} A(\lambda)^{-1} A_{1} d \lambda=Z_{m-1} A_{1}, \\
P_{\mathscr{N}^{\prime}, \mathscr{R}^{\prime}} & =\frac{1}{2 \pi i} \int_{\partial D_{\rho}} A_{1} A(\lambda)^{-1} d \lambda=A_{1} Z_{m-1},
\end{aligned}
$$


where $\partial D_{\rho}$ is the boundary of $D_{\rho}$. That is, $P_{\mathscr{N}, \mathscr{R}}$ coincides with the right logarithmic residue of $A(\lambda)$ at 0 and $P_{\mathscr{N}^{\prime}, \mathscr{R}^{\prime}}$ with the left logarithmic residue of $A(\lambda)$ at 0 , see [7-9] and references therein for general results on logarithmic residues and idempotents.

The results of the present paper are the following: first, necessary and sufficient conditions for a pole of order $m$ are given; these conditions are expressed in terms of certain orthogonal direct sum decompositions

$$
X=\bigoplus_{h=0}^{m} \beta_{h}, \quad \beta_{0}, \beta_{m} \neq 0, \quad Y=\bigoplus_{h=0}^{m} \alpha_{h}, \quad \alpha_{0}, \alpha_{m} \neq 0,
$$

where $\beta_{h}$ and $\alpha_{h}$ are closed subspaces defined recursively by the 'local subspace decomposition', see Definition 2.2 below. This recursion employs the first $m+1$ fundamental equations, i.e.

$$
A_{0} Z_{n}+A_{1} Z_{n-1}=1_{n=m} I, \quad Z_{n} A_{0}+Z_{n-1} A_{1}=1_{n=m} I, \quad n=0, \ldots, m,
$$

and defines certain operators $S_{h}$ whose images and orthogonal complements of the null spaces, $\alpha_{h}=\operatorname{Im} S_{h}$ and $\beta_{h}=\left(\operatorname{Ker} S_{h}\right)^{\perp}$, deliver the orthogonal direct sum decompositions that characterize the order of the pole.

The same recursion defines operators $M_{\beta} \in \mathscr{B}_{X}$ and $M_{\alpha} \in \mathscr{B}_{Y}$ that allow to construct the factors in

$$
A(\lambda)=E(\lambda) D(\lambda) F(\lambda)
$$

where $E(\lambda), F(\lambda)$ are analytic and invertible on $D_{\rho}$ and $D(\lambda)$ describes the singularity of $A(\lambda)$ at 0 , see Gohberg and Sigal [17]. This in turn delivers chains that allow to construct the subspaces in (1.6) as

$$
\begin{array}{ll}
\mathscr{N}_{m}=\bigoplus_{h=1}^{m} \bigoplus_{k=0}^{h-1} M_{\beta}^{k} \beta_{h}, & \mathscr{R}_{m}=\left(A_{1}^{*} \bigoplus_{h=1}^{m} \bigoplus_{k=0}^{h-1} M_{\alpha}^{k} \alpha_{h}\right)^{\perp}, \\
\mathscr{N}_{m}^{\prime}=A_{1} \bigoplus_{h=1}^{m} \bigoplus_{k=0}^{h-1} M_{\beta}^{k} \beta_{h}, & \mathscr{R}_{m}^{\prime}=\left(\bigoplus_{h=1}^{m} \bigoplus_{k=0}^{h-1} M_{\alpha}^{k} \alpha_{h}\right)^{\perp} .
\end{array}
$$

This allows to compute the operators $Z_{n \mathscr{N}^{\prime}}, Z_{n \mathscr{R}^{\prime}}$ and the projections $P_{\mathscr{N}, \mathscr{R}}, P_{\mathscr{R}, \mathscr{N}}$, $P_{\mathscr{N}^{\prime}, \mathscr{R}^{\prime}}, P_{\mathscr{R}^{\prime}, \mathscr{N}^{\prime}}$, i.e. it provides a construction of the resolvent and of the logarithmic residues in terms of the quantities defined by the local subspace decomposition.

Further it is shown that the same quantities characterize image and null space of each operator in the principal part of the resolvent, i.e.

$$
\operatorname{Im} Z_{n}=\bigoplus_{h=m-n}^{m} \bigoplus_{k=0}^{h-m+n} M_{\beta}^{k} \beta_{h}, \quad \operatorname{Ker} Z_{n}=\left(\bigoplus_{h=m-n}^{m} \bigoplus_{k=0}^{h-m+n} M_{\alpha}^{k} \alpha_{h}\right)^{\perp}
$$


where $n=0, \ldots, m-1$.

The last part of the present paper considers the special case of $A_{0}$ being Fredholm of index 0 ; it characterizes the rank of the operators in the principal part of the resolvent and links the dimensions of the subspaces in (1.6) to the partial multiplicities and to the algebraic multiplicity of $A(\lambda)$ at 0 .

The rest of the paper is organized as follows: the remaining of this section reports notational conventions and preliminaries, Section 2 presents definitions and main results and Section 3 illustrates some of the results for the particular cases of poles of order 1 and 2. Section 4 considers a pole of order $m \in \mathbb{N}$ and corresponding factorizations of $A(\lambda)$, Section 5 describes chains and the associated subspaces that characterize the principal part of the resolvent and the logarithmic residues and Section 6 considers the particular case of $A_{0}$ being Fredholm of index 0. Appendix A contains proofs.

\subsection{Notation and preliminaries}

$\mathscr{B}_{X, Y}$ denotes the space of bounded linear mappings between two complex Hilbert spaces $X$ and $Y$ (when $X=Y$, the shorthand notation $\mathscr{B}_{X}=\mathscr{B}_{X, X}$ is employed). Image and null space of $T \in \mathscr{B}_{X, Y}$ are respectively denoted by $\operatorname{Im} T=\{y \in Y: y=$ $T x, x \in X\}$ and $\operatorname{Ker} T=\{x \in X: T x=0\}$. Note that $\operatorname{Ker} T$ is closed while $\operatorname{Im} T$ may not be so. $T^{*} \in \mathscr{B}_{Y, X}$ denotes the adjoint of $T$; recall that $\operatorname{Ker} T=\left(\operatorname{Im} T^{*}\right)^{\perp}$, $\operatorname{Ker} T^{*}=(\operatorname{Im} T)^{\perp}$ and if $\operatorname{Im} T$ is closed, $\operatorname{Im} T=\left(\operatorname{Ker} T^{*}\right)^{\perp}, \operatorname{Im} T^{*}=(\operatorname{Ker} T)^{\perp}$. If $\operatorname{Im} T$ is closed, its generalized inverse $T^{+} \in \mathscr{B}_{Y, X}$ exists and it is unique; recall that $T^{+} T T^{+}=T^{+}, T T^{+} T=T, T T^{+}=P_{\operatorname{Im} T}, T^{+} T=P_{(\operatorname{Ker} T)^{\perp}}, \operatorname{Ker} T^{+}=(\operatorname{Im} T)^{\perp}$, and $\operatorname{Im} T^{+}=(\operatorname{Ker} T)^{\perp}$. A Hilbert space $Z$ is said to be the direct sum of $\zeta$ and $\varphi$, written $Z=\zeta \oplus \varphi$, if $(i) Z=\zeta+\varphi=\{u+v: u \in \zeta, v \in \varphi\}$ and $(i i) \zeta \cap \varphi=0$. If $\zeta$ and $\varphi$ are closed subspaces and $Z=\zeta \oplus \varphi$, one has the associated projection identity $I=P_{\zeta, \varphi}+P_{\varphi, \zeta}$, where $P_{\zeta, \varphi} \in \mathscr{B}_{Z}$ denotes the projection of $Z$ onto $\zeta$ along $\varphi$, i.e. $P_{\zeta, \varphi}=P_{\zeta, \varphi}^{2}, \operatorname{Im} P_{\zeta, \varphi}=\zeta$ and $\operatorname{Ker} P_{\zeta, \varphi}=\varphi$; similarly for $P_{\varphi, \zeta}$. When $\varphi=\zeta^{\perp}$, the direct sum is said to be orthogonal and the shorthand notation $P_{\zeta}=P_{\zeta, \zeta^{\perp}}$ is employed for the orthogonal projection onto $\zeta$; similarly for $P_{\zeta^{\perp}}$.

\section{Definitions and main results}

This section introduces the local subspace decomposition and presents the main results. The analysis is conducted under the following assumption.

Assumption 2.1 ( $\operatorname{Im} A_{0}$ is closed and $A_{0}$ is singular). Assume that $(i) \operatorname{Im} A_{0}$ is closed and $(i i) A_{0} \neq 0$ is neither one-to-one nor onto.

Note that, under Assumption 2.1, $\operatorname{Im} A_{0},\left(\operatorname{Im} A_{0}\right)^{\perp}, \operatorname{Ker} A_{0},\left(\operatorname{Ker} A_{0}\right)^{\perp}$ are all nonzero closed subspaces. Hence one has the orthogonal direct sum decompositions 


$$
\begin{array}{lll}
Y=\alpha_{0} \oplus \alpha_{0}^{\perp}, & \alpha_{0}, \alpha_{0}^{\perp} \neq 0, & \alpha_{0}=\operatorname{Im} A_{0}, \\
X=\beta_{0} \oplus \beta_{0}^{\perp}, & \beta_{0}, \beta_{0}^{\perp} \neq 0, & \beta_{0}=\left(\operatorname{Ker} A_{0}\right)^{\perp},
\end{array}
$$

and the associated orthogonal projections identities

$$
\begin{array}{lll}
P_{\alpha_{0}}+P_{\alpha_{0}^{\perp}}=I, & P_{\alpha_{0}}, P_{\alpha_{0}^{\perp}} \in \mathscr{B}_{Y}, & P_{\alpha_{0}}, P_{\alpha_{0}^{\perp}} \neq 0, \\
P_{\beta_{0}}+P_{\beta_{0}^{\perp}}=I, & P_{\beta_{0}}, P_{\beta_{0}^{\perp}} \in \mathscr{B}_{X}, & P_{\beta_{0}}, P_{\beta_{0}^{\perp}} \neq 0,
\end{array}
$$

where

$$
\begin{array}{ll}
\operatorname{Im} P_{\alpha_{0}}=\operatorname{Ker} P_{\alpha_{0}^{\perp}}=\alpha_{0}, & \operatorname{Ker} P_{\alpha_{0}}=\operatorname{Im} P_{\alpha_{0}^{\perp}}=\alpha_{0}^{\perp}, \\
\operatorname{Im} P_{\beta_{0}}=\operatorname{Ker} P_{\beta_{0}^{\perp}}=\beta_{0}, & \operatorname{Ker} P_{\beta_{0}}=\operatorname{Im} P_{\beta_{0}^{\perp}}=\beta_{0}^{\perp} .
\end{array}
$$

Moreover, the generalized inverse $A_{0}^{+}$of $A_{0}$ is such that

$$
A_{0} A_{0}^{+}=P_{\alpha_{0}}, \quad A_{0}^{+} A_{0}=P_{\beta_{0}}
$$

Definition 2.2 (Local subspace decomposition). Let $m \in \mathbb{N}$,

$$
S_{0}=A_{0}, \quad \alpha_{0}=\operatorname{Im} S_{0}, \quad \beta_{0}=\left(\operatorname{Ker} S_{0}\right)^{\perp},
$$

and for $h=1, \ldots, m$ define

$$
S_{h}=P_{a_{h}^{\perp}} Q_{h} P_{b_{h}^{\perp}}, \quad \alpha_{h}=\operatorname{Im} S_{h}, \quad \beta_{h}=\left(\operatorname{Ker} S_{h}\right)^{\perp},
$$

where

$$
a_{h}=\bigoplus_{j=0}^{h-1} \alpha_{j}, \quad b_{h}=\bigoplus_{j=0}^{h-1} \beta_{j}, \quad Q_{h}=\left\{\begin{array}{cc}
A_{1}, & h=1 \\
-Q_{h-1} \sum_{j=0}^{h-2} S_{j}^{+} Q_{j+1}, & h=2, \ldots, m
\end{array}\right.
$$

Further let

$$
M_{\beta}=-\sum_{h=0}^{m-1} S_{h}^{+} Q_{h+1}, \quad M_{\alpha}=\left(-\sum_{h=0}^{m-1} Q_{h+1} S_{h}^{+}\right)^{*}
$$

Note that

$$
\alpha_{h} \subseteq a_{h}^{\perp}=\left(\alpha_{0} \oplus \cdots \oplus \alpha_{h-1}\right)^{\perp}, \quad \beta_{h} \subseteq b_{h}^{\perp}=\left(\beta_{0} \oplus \cdots \oplus \beta_{h-1}\right)^{\perp}, \quad h=1, \ldots, m
$$

i.e. $\alpha_{h}$ is orthogonal to $\alpha_{j}$ and $\beta_{h}$ is orthogonal to $\beta_{j}, h \neq j$. It turns out that for $h \neq 0, m$, it is possible that $\alpha_{h}=0$ and $\beta_{h}=0$. In what follows, every statement concerning $\alpha_{h}$ or $\beta_{h}$ implicitly assumes that they are nonzero; the modifications required otherwise are straightforward. 
Observe that $\beta_{h}=\left(\operatorname{Ker} S_{h}\right)^{\perp}, \beta_{h}^{\perp}=\operatorname{Ker} S_{h}$ and $\alpha_{h}^{\perp}=\left(\operatorname{Im} S_{h}\right)^{\perp}$ are closed subspaces and also $\alpha_{h}=\operatorname{Im} S_{h}$ need to be so. Indeed, let $\xi_{h}$ be the orthogonal complement of $\alpha_{h}$ in $a_{h}^{\perp}$, i.e. $a_{h}^{\perp}=\alpha_{h} \oplus \xi_{h}$; because the closed subspace $\xi_{h}$ complements the image $\alpha_{h}$ of the bounded operator $S_{h}$ in the closed subspace $a_{h}^{\perp}$, it follows from Theorem IV.1.12 in [18] that $\alpha_{h}=\operatorname{Im} S_{h}$ must be closed.

Hence $a_{h}, a_{h}^{\perp}, b_{h}, b_{h}^{\perp}, h=1, \ldots, m$, are all nonzero closed subspaces and one has the orthogonal direct sum decompositions

$$
\begin{aligned}
Y=a_{h} \oplus a_{h}^{\perp}, & a_{h}, a_{h}^{\perp} \neq 0, & a_{h}=\bigoplus_{j=0}^{h-1} \alpha_{j}, \\
X=b_{h} \oplus b_{h}^{\perp}, & b_{h}, b_{h}^{\perp} \neq 0, & b_{h}=\bigoplus_{j=0}^{h-1} \beta_{j},
\end{aligned}
$$

and the associated orthogonal projections identities

$$
\begin{array}{lll}
P_{a_{h}}+P_{a_{h}^{\perp}}=I, & P_{a_{h}}, P_{a_{h}^{\perp}} \in \mathscr{B}_{Y}, & P_{a_{h}}, P_{a_{h}^{\perp}} \neq 0, \\
P_{b_{h}}+P_{b_{h}^{\perp}}=I, & P_{b_{h}}, P_{b_{h}^{\perp}} \in \mathscr{B}_{X}, & P_{b_{h}}, P_{b_{h}^{\perp}} \neq 0 .
\end{array}
$$

Moreover,

$$
\begin{array}{rlrl}
S_{h} S_{h}^{+} & =P_{\alpha_{h}}, & S_{h}^{+} S_{h} & =P_{\beta_{h}}, \\
\operatorname{Ker} S_{h}^{+} & =\alpha_{h}^{\perp}, & & \operatorname{Im} S_{h}^{+}=\beta_{h}, \\
S_{h}^{+} P_{a_{h}^{\perp}}=S_{h}^{+}, & P_{b_{h}^{\perp}} S_{h}^{+}=S_{h}^{+} .
\end{array}
$$

The first result links the order of the pole of the resolvent to the $\alpha_{h}$ and $\beta_{h}$ subspaces; further it shows that $Z_{0}$ is the generalized inverse of $S_{m}$, i.e. $Z_{0}=S_{m}^{+}$.

Theorem 2.3 (Pole of order $m$ ). The following statements are equivalent:

(i) $A(\lambda)^{-1}$ has a pole of order $m \geq 1$ at 0 ,

(ii) $X=\bigoplus_{h=0}^{m} \beta_{h}$, where $\beta_{h}$ is closed and $\beta_{0}, \beta_{m} \neq 0$,

(iii) $Y=\bigoplus_{h=0}^{m} \alpha_{h}$, where $\alpha_{h}$ is closed and $\alpha_{0}, \alpha_{m} \neq 0$,

(iv) $Z_{0}=S_{m}^{+}$.

The second result provides two alternative constructions of the factorization $A(\lambda)=$ $E(\lambda) D(\lambda) F(\lambda)$, where $E(\lambda), F(\lambda)$ are analytic and invertible on $D_{\rho}$ and $D(\lambda)$ describes the singularity of $A(\lambda)$ at 0 .

Theorem 2.4 (Factorizations). Consider Definition 2.2 and let

$$
E_{\beta}(\lambda)=\sum_{n=0}^{\infty} E_{\beta, n} \lambda^{h}, \quad D_{\beta}(\lambda)=\sum_{h=0}^{m} P_{\beta_{h}} \lambda^{h}, \quad F_{\beta}(\lambda)=I-M_{\beta} \lambda
$$




$$
E_{\alpha}(\lambda)=I-M_{\alpha}^{*} \lambda, \quad D_{\alpha}(\lambda)=\sum_{h=0}^{m} P_{\alpha_{h}} \lambda^{h}, \quad F_{\alpha}(\lambda)=\sum_{n=0}^{\infty} F_{\alpha, n} \lambda^{h}
$$

where

$$
E_{\beta, 0}=F_{\alpha, 0}=\sum_{h=0}^{m} S_{h}, \quad \begin{aligned}
& E_{\beta, n}=P_{\alpha \perp} A_{1} M_{\beta}^{n-1} B_{\beta, m}, \\
& F_{\alpha, n}=C_{\alpha, m}\left(M_{\alpha}^{*}\right)^{n-1} A_{1} P_{\beta_{0}^{\perp}}
\end{aligned}, \quad n=1,2, \ldots
$$

and

$$
B_{\beta, n}=\sum_{k=0}^{n} M_{\beta}^{k} P_{\beta_{m-n+k}}, \quad C_{\alpha, n}=\sum_{k=0}^{n} P_{\alpha_{m-n+k}}\left(M_{\alpha}^{*}\right)^{k}, \quad n=0, \ldots, m
$$

Then

$$
A(\lambda)=E_{\zeta}(\lambda) D_{\zeta}(\lambda) F_{\zeta}(\lambda), \quad \zeta=\alpha, \beta,
$$

where $E_{\zeta}(\lambda), F_{\zeta}(\lambda)$ are analytic and invertible on $D_{\rho}$.

This in turn allows to compute the resolvent and the logarithmic residues, see Corollary 4.3 below.

The third result links these results with the classical ones based on ascent and descent in Bart and Lay [10], showing how the subspaces in (1.6) can be constructed using the quantities defined by the local subspace decomposition.

Theorem $2.5\left(\mathscr{N}_{m}, \mathscr{R}_{m}, \mathscr{N}_{m}^{\prime}, \mathscr{R}_{m}^{\prime}\right)$. The subspaces in (1.6) can be constructed as

$$
\begin{array}{ll}
\mathscr{N}_{m}=\bigoplus_{h=1}^{m} \bigoplus_{k=0}^{h-1} M_{\beta}^{k} \beta_{h}, & \mathscr{R}_{m}=\left(A_{1}^{*} \bigoplus_{h=1}^{m} \bigoplus_{k=0}^{h-1} M_{\alpha}^{k} \alpha_{h}\right)^{\perp}, \\
\mathscr{N}_{m}^{\prime}=A_{1} \bigoplus_{h=1}^{m} \bigoplus_{k=0}^{h-1} M_{\beta}^{k} \beta_{h}, & \mathscr{R}_{m}^{\prime}=\left(\bigoplus_{h=1}^{m} \bigoplus_{k=0}^{h-1} M_{\alpha}^{k} \alpha_{h}\right)^{\perp} .
\end{array}
$$

One can thus use these formulas to compute the restricted operators $Z_{n \mathscr{N}^{\prime}}, Z_{n \mathscr{R}^{\prime}}$ and the projections which deliver the resolvent and the logarithmic residues.

The next result characterizes image and null space of each operator in the principal part of the resolvent.

Theorem 2.6 (Image and null space of $Z_{n}, n=0, \ldots, m-1$ ). For $n=0, \ldots, m-1$, image and null space of $Z_{n}$ are given by

$$
\operatorname{Im} Z_{n}=\bigoplus_{h=m-n}^{m} \bigoplus_{k=0}^{h-m+n} M_{\beta}^{k} \beta_{h}, \quad \operatorname{Ker} Z_{n}=\left(\bigoplus_{h=m-n}^{m} \bigoplus_{k=0}^{h-m+n} M_{\alpha}^{k} \alpha_{h}\right)^{\perp}
$$


The last part of the present paper considers the special case of $A_{0}$ being Fredholm of index 0 ; it characterizes the rank of the operators in the principal part of the resolvent and links the dimensions of the subspaces in (1.6) to the partial multiplicities and to the algebraic multiplicity of $A(\lambda)$ at 0 .

Theorem 2.7 (Special case: $A_{0}$ is Fredholm of index 0). If $A_{0}$ is Fredholm of index 0, let $r_{h}=\operatorname{dim} \alpha_{h}=\operatorname{dim} \beta_{h}, h=1, \ldots, m$. Then

(i) $0 \leq r_{h}<\infty, h=1, \ldots, m-1$, and $0<r_{m}<\infty$,

(ii) $\operatorname{rank} Z_{n}=\sum_{h=0}^{n}(h+1) r_{m-n+h}<\infty, n=0, \ldots, m-1$,

(iii) $\operatorname{dim} \mathscr{N}_{m}=\operatorname{dim} \mathscr{N}_{m}^{\prime}=\sum_{h=1}^{m} h r_{h}<\infty$,

(iv) the algebraic multiplicity of $A(\lambda)$ at 0 is equal to $\sum_{h=1}^{m} h r_{h}<\infty$,

$(v)$ the number of distinct partial multiplicities of $A(\lambda)$ at 0 is equal to $\sum_{h=1}^{m} 1_{r_{h}>0}$,

(vi) there are exactly $r_{h}$ partial multiplicities that are equal to $h=1, \ldots, m$.

\section{Poles of order 1 and 2}

This section illustrates the main ideas behind the local subspace decomposition considering poles of order 1 and 2. The results in the present section are thus particular cases of those in Section 2 but direct proofs are given here in order to illustrate the working of the general case described in Section 4.

In the following, equations in system (1.8) are labelled according to the value of $n$; for instance $A_{0} Z_{0}=0$ and $Z_{0} A_{0}=0$ are equations 0 and the identities appear in equations $m$, which is the order of the pole. The equations derived from $A(\lambda) A(\lambda)^{-1}=I \in \mathscr{B}_{Y}$ are called $Y$ versions (and correspond to the first equation in (1.8)) and those that derive from $A(\lambda)^{-1} A(\lambda)=I \in \mathscr{B}_{X}$ are called $X$ versions (and correspond to the second equation in (1.8)); for instance $A_{0} Z_{m}+A_{1} Z_{m-1}=I$ is the $Y$ version of equation $m$.

Some implications of equations 0 and 1 are next derived; remark that these properties hold for any value of $m$. From equations $0, A_{0} Z_{0}=0$ and $Z_{0} A_{0}=0$, one respectively has

$$
\operatorname{Im} Z_{0} \subseteq \operatorname{Ker} A_{0}, \quad \operatorname{Ker} Z_{0} \supseteq \operatorname{Im} A_{0},
$$

i.e., letting $\beta_{0}=\left(\operatorname{Ker} A_{0}\right)^{\perp}$ and $\alpha_{0}=\operatorname{Im} A_{0}$, one has

$$
\operatorname{Im} Z_{0} \subseteq \beta_{0}^{\perp}, \quad \operatorname{Ker} Z_{0} \supseteq \alpha_{0}
$$

The 1.h.s. of the $Y$ version of equation 1 reads $A_{0} Z_{1}+A_{1} Z_{0}$; use $P_{\beta_{0}^{\perp}}+P_{\beta_{0}}=I$ to write $A_{1} Z_{0}=A_{1}\left(P_{\beta_{0}^{\perp}}+P_{\beta_{0}}\right) Z_{0}=A_{1} P_{\beta_{0}^{\perp}} Z_{0}+A_{1} P_{\beta_{0}} Z_{0}=A_{1} P_{\beta_{0}^{\perp}} Z_{0}$, because $P_{\beta_{0}} Z_{0}=0$ follows from $\operatorname{Im} Z_{0} \subseteq \beta_{0}^{\perp}$. Applying $P_{\alpha_{0}^{\perp}}$ on both sides of $A_{1} Z_{0}=A_{1} P_{\beta_{0}^{\perp}} Z_{0}$ one has

$$
P_{\alpha_{0}^{\perp}} A_{1} Z_{0}=S_{1} Z_{0}
$$

having defined $S_{1}=P_{\alpha_{0}^{\perp}} A_{1} P_{\beta_{0}^{\perp}}$; note that, by definition, 


$$
\operatorname{Im} S_{1} \subseteq \operatorname{Im} P_{\alpha_{0}^{\perp}}=\alpha_{0}^{\perp}, \quad \operatorname{Ker} S_{1} \supseteq \operatorname{Ker} P_{\beta_{0}^{\perp}}=\beta_{0}
$$

i.e., letting $\alpha_{1}=\operatorname{Im} S_{1}$ and $\beta_{1}=\left(\operatorname{Ker} S_{1}\right)^{\perp}$, one has

$$
\alpha_{1} \subseteq \alpha_{0}^{\perp}, \quad \beta_{1} \subseteq \beta_{0}^{\perp}
$$

This shows that from the l.h.s. of the $Y$ version of equation $1, A_{0} Z_{1}+A_{1} Z_{0}$, one has

$$
P_{\alpha_{0}^{\perp}}\left(A_{0} Z_{1}+A_{1} Z_{0}\right)=S_{1} Z_{0}
$$

because $P_{\alpha_{0}^{\perp}} A_{0}=0$ follows from $\operatorname{Im} A_{0}=\alpha_{0}$.

Similarly, starting from the l.h.s. of the $X$ version of equation $1, Z_{1} A_{0}+Z_{0} A_{1}$, and using $P_{\alpha_{0}}+P_{\alpha_{0}^{\perp}}=I$ one has $Z_{0} A_{1}=Z_{0}\left(P_{\alpha_{0}^{\perp}}+P_{\alpha_{0}}\right) A_{1}=Z_{0} P_{\alpha_{0}^{\perp}} A_{1}+Z_{0} P_{\alpha_{0}} A_{1}=$ $Z_{0} P_{\alpha_{0}^{\perp}} A_{1}$, because $Z_{0} P_{\alpha_{0}}$ follows from Ker $Z_{0} \supseteq \alpha_{0}$. Hence $Z_{0} A_{1} P_{\beta_{0}^{\perp}}=Z_{0} P_{\alpha_{0}^{\perp}} A_{1} P_{\beta_{0}^{\perp}}$, i.e. $Z_{0} A_{1} P_{\beta_{0}^{\perp}}=Z_{0} S_{1}$. This shows that from the l.h.s. of the $X$ version of equation 1 one has

$$
\left(Z_{1} A_{0}+Z_{0} A_{1}\right) P_{\beta_{0}^{\perp}}=Z_{0} S_{1}
$$

because $A_{0} P_{\beta_{0}^{\perp}}=0$ follows from $\operatorname{Ker} A_{0}=\beta_{0}^{\perp}$.

\subsection{Poles of order 1}

Consider the case in which $A(\lambda)^{-1}$ has a pole of order $m=1$ at 0. In this case, (1.5) reads

$$
A(\lambda)^{-1}=\frac{Z_{0}}{\lambda}+\sum_{n=1}^{\infty} Z_{n} \lambda^{n-1}, \quad \lambda \in D_{\rho} \backslash\{0\}, \quad Z_{0} \neq 0
$$

and (1.8) reads

$$
\begin{aligned}
A_{0} Z_{0} & =0, & Z_{0} A_{0} & =0, \\
A_{0} Z_{1}+A_{1} Z_{0} & =I, & Z_{1} A_{0}+Z_{0} A_{1} & =I .
\end{aligned}
$$

Because the identity is in equations 1, (3.1) and (3.2) respectively imply

$$
S_{1} Z_{0}=P_{\alpha_{0}^{\perp}}, \quad Z_{0} S_{1}=P_{\beta_{0}^{\perp}}
$$

Recall that $\alpha_{0} \subseteq \operatorname{Ker} Z_{0}$ and $\operatorname{Im} S_{1}=\alpha_{1} \subseteq \alpha_{0}^{\perp}$; from $S_{1} Z_{0}=P_{\alpha_{0}^{\perp}}$ one has

$$
\operatorname{Ker} Z_{0}=\operatorname{Ker} P_{\alpha_{0}^{\perp}}=\alpha_{0}, \quad \operatorname{Im} S_{1}=\operatorname{Im} P_{\alpha_{0}^{\perp}}=\alpha_{0}^{\perp} \text {, }
$$

and recalling that $\operatorname{Im} Z_{0} \subseteq \beta_{0}^{\perp}$ and Ker $S_{1}=\beta_{1}^{\perp} \supseteq \beta_{0}$, from $Z_{0} S_{1}=P_{\beta_{0}^{\perp}}$ one finds 


$$
\operatorname{Im} Z_{0}=\operatorname{Im} P_{\beta_{0}^{\perp}}=\beta_{0}^{\perp}, \quad \operatorname{Ker} S_{1}=\operatorname{Ker} P_{\beta_{0}^{\perp}}=\beta_{0}
$$

This shows that if $m=1$ then

$$
\alpha_{1}=\alpha_{0}^{\perp}, \quad \beta_{1}=\beta_{0}^{\perp},
$$

i.e.

$$
X=\beta_{0} \oplus \beta_{1}, \quad Y=\alpha_{0} \oplus \alpha_{1}
$$

Conversely, when $m>1$ the identity is not in equations 1 and (3.1) and (3.2) respectively imply

$$
S_{1} Z_{0}=0, \quad Z_{0} S_{1}=0
$$

From $A_{0} Z_{0}=0$ and $S_{1} Z_{0}=0$ one has

$$
\operatorname{Im} Z_{0} \subseteq\left(\operatorname{Ker} A_{0} \cap \operatorname{Ker} S_{1}\right)=\beta_{0}^{\perp} \cap \beta_{1}^{\perp}=\left(\beta_{0} \oplus \beta_{1}\right)^{\perp}
$$

Recall that $\beta_{1} \subseteq \beta_{0}^{\perp}$; because $\beta_{1}=\beta_{0}^{\perp}$ implies $\operatorname{Im} Z_{0}=0$, if $m>1$ then $\beta_{1} \subset \beta_{0}^{\perp}$. Thus $\beta_{1}=\beta_{0}^{\perp}$ holds if and only if $m=1$.

Similarly, from $Z_{0} A_{0}=0$ and $Z_{0} S_{1}=0$ one has

$$
\operatorname{Ker} Z_{0} \supseteq\left(\operatorname{Im} A_{0} \oplus \operatorname{Im} S_{1}\right)=\alpha_{0} \oplus \alpha_{1} \text {. }
$$

Recall that $\alpha_{1} \subseteq \alpha_{0}^{\perp}$; because $\alpha_{1}=\alpha_{0}^{\perp}$ implies Ker $Z_{0}=Y$, if $m>1$ then $\alpha_{1} \subset \alpha_{0}^{\perp}$. Thus $\alpha_{1}=\alpha_{0}^{\perp}$ holds if and only if $m=1$.

When $m=1$, (3.3) thus reads $S_{1} Z_{0}=P_{\alpha_{1}}$ and $Z_{0} S_{1}=P_{\beta_{1}}$. The former implies $Z_{0} S_{1} Z_{0}=Z_{0} P_{\alpha_{1}}$ and because $Z_{0}=Z_{0} P_{\alpha_{1}}$ one finds $Z_{0} S_{1} Z_{0}=Z_{0}$; similarly, from the latter one has $S_{1} Z_{0} S_{1}=S_{1} P_{\beta_{1}}$ and because $S_{1}=S_{1} P_{\beta_{1}}$ one finds $S_{1} Z_{0} S_{1}=S_{1}$. This shows that, when the pole has order one, $Z_{0}$ is the generalized inverse of $S_{1}$.

This proves the following statement.

Proposition 3.1 (Pole of order one). Let

$$
\begin{array}{lll}
S_{0}=A_{0}, & \alpha_{0}=\operatorname{Im} S_{0}, & \beta_{0}=\left(\operatorname{Ker} S_{0}\right)^{\perp}, \\
S_{1}=P_{\alpha_{0}^{\perp}} Q_{1} P_{\beta_{0}^{\perp}}, & \alpha_{1}=\operatorname{Im} S_{1}, & \beta_{1}=\left(\operatorname{Ker} S_{1}\right)^{\perp}
\end{array}
$$

where $Q_{1}=A_{1}$. The following statements are equivalent:

(i) $A(\lambda)^{-1}$ has a pole of order $m=1$ at 0 ,

(ii) $X=\beta_{0} \oplus \beta_{1}$, where $\beta_{h}$ is closed and $\beta_{0}, \beta_{1} \neq 0$,

(iii) $Y=\alpha_{0} \oplus \alpha_{1}$, where $\alpha_{h}$ is closed and $\alpha_{0}, \alpha_{1} \neq 0$,

(iv) $Z_{0}=S_{1}^{+}$. 
Thus, when the pole has order one, $Z_{0}$ is such that

$$
\operatorname{Im} Z_{0}=\beta_{1}, \quad \operatorname{Ker} Z_{0}=\alpha_{0}
$$

Observe that these quantities are all expressed in terms of $A_{0}$ and $A_{1}$ via the definitions in (3.6) and (3.7); further note that Proposition 3.1 is found by setting $m=1$ in Theorem 2.3.

Next we illustrate the derivation of the $\beta$ factorization $A(\lambda)=E_{\beta}(\lambda) D_{\beta}(\lambda) F_{\beta}(\lambda)$ in Theorem 2.4 for $m=1$ (the $\alpha$ factorization is obtained similarly from the $X$ versions of the fundamental equations). From the above one has

$$
P_{\beta_{0}} Z_{0}=0, \quad P_{\beta_{1}} Z_{0}=S_{1}^{+}
$$

and from $A_{0} Z_{1}+A_{1} Z_{0}=I$ it follows that $A_{0}^{+} A_{0} Z_{1}+A_{0}^{+} A_{1} Z_{0}=A_{0}^{+}$; substituting $A_{0}^{+} A_{0}=P_{\beta_{0}}$, see $(2.1)$, and rearranging one finds

$$
P_{\beta_{0}} Z_{1}=S_{0}^{+}-S_{0}^{+} Q_{1} Z_{0}, \quad S_{0}=A_{0}, \quad Q_{1}=A_{1} .
$$

Hence

$$
\begin{gathered}
P_{\beta_{0}} A(\lambda)^{-1}=\frac{P_{\beta_{0}} Z_{0}}{\lambda}+\sum_{n=1}^{\infty} P_{\beta_{0}} Z_{n} \lambda^{n-1}=P_{\beta_{0}} Z_{1}+\sum_{n=2}^{\infty} P_{\beta_{0}} Z_{n} \lambda^{n-1} \\
=S_{0}^{+}-S_{0}^{+} Q_{1} Z_{0}+\sum_{n=1}^{\infty} P_{\beta_{0}} Z_{n+1} \lambda^{n} .
\end{gathered}
$$

Applying $\lambda S_{0}^{+} Q_{1}$ to $A(\lambda)^{-1}$ one has

$$
\left(\lambda S_{0}^{+} Q_{1}\right) A(\lambda)^{-1}=S_{0}^{+} Q_{1} Z_{0}+\sum_{n=1}^{\infty} S_{0}^{+} Q_{1} Z_{n} \lambda^{n}
$$

and summing up the two expressions one finds

$$
\left(P_{\beta_{0}}+\lambda S_{0}^{+} Q_{1}\right) A(\lambda)^{-1}=S_{0}^{+}+\sum_{n=1}^{\infty}\left(P_{\beta_{0}} Z_{n+1}+S_{0}^{+} Q_{1} Z_{n}\right) \lambda^{n}
$$

i.e., see Proposition 4.2 below,

$$
F_{0}(\lambda) A(\lambda)^{-1}=\widetilde{F}_{0}(\lambda)
$$

where $F_{0}(\lambda)=P_{\beta_{0}}+\lambda S_{0}^{+} Q_{1}, \widetilde{F}_{0}(\lambda)=S_{0}^{+}+\sum_{n=1}^{\infty}\left(P_{\beta_{0}} Z_{n+1}+S_{0}^{+} Q_{1} Z_{n}\right) \lambda^{n}$ is analytic on $D_{\rho}$ and $\widetilde{F}_{0}(0)=S_{0}^{+} \neq 0$. Moreover, 


$$
P_{\beta_{1}} A(\lambda)^{-1}=\frac{P_{\beta_{1}} Z_{0}}{\lambda}+\sum_{n=1}^{\infty} P_{\beta_{1}} Z_{n} \lambda^{n-1}=\frac{S_{1}^{+}}{\lambda}+\sum_{n=1}^{\infty} P_{\beta_{1}} Z_{n} \lambda^{n-1}
$$

i.e., see Proposition 4.2 below,

$$
F_{1}(\lambda) A(\lambda)^{-1}=\lambda^{-1} \widetilde{F}_{1}(\lambda)
$$

where $F_{1}(\lambda)=P_{\beta_{1}}, \widetilde{F}_{1}(\lambda)=S_{1}^{+}+\sum_{n=1}^{\infty} P_{\beta_{1}} Z_{n} \lambda^{n}$ is analytic on $D_{\rho}$ and $\widetilde{F}_{1}(0)=S_{1}^{+} \neq 0$. Hence one has

$$
\widetilde{F}_{h}(\lambda) A(\lambda)=\lambda^{h} F_{h}(\lambda), \quad \widetilde{F}_{0}(\lambda)=S_{h}^{+}, \quad h=0,1,
$$

where $\operatorname{Im} S_{h}^{+}=\beta_{h}$ and $\operatorname{Ker} S_{h}^{+}=\alpha_{h}^{\perp}$, see (2.1). Summing over $h$ one finds

$$
\widetilde{F}(\lambda) A(\lambda)=\sum_{h=0}^{1} \lambda^{h} F_{h}(\lambda), \quad \widetilde{F}(\lambda)=\sum_{h=0}^{1} \widetilde{F}_{h}(\lambda)
$$

where $\widetilde{F}(\lambda)$ is analytic on $D_{\rho}, \widetilde{F}(0)=\sum_{h=0}^{1} S_{h}^{+}$is invertible and

$$
\sum_{h=0}^{1} \lambda^{h} F_{h}(\lambda)=P_{\beta_{0}}+\lambda\left(P_{\beta_{1}}+S_{0}^{+} Q_{1}\right)
$$

On the other hand, letting

$$
D_{\beta}(\lambda)=P_{\beta_{0}}+P_{\beta_{1}} \lambda, \quad F_{\beta}(\lambda)=I-M_{\beta} \lambda, \quad M_{\beta}=-S_{0}^{+} Q_{1},
$$

one has $P_{\beta_{0}} M_{\beta}=-P_{\beta_{0}} S_{0}^{+} Q_{1}=-S_{0}^{+} Q_{1}$ and $P_{\beta_{1}} M_{\beta}=-P_{\beta_{1}} S_{0}^{+} Q_{1}=0$ and hence

$$
\begin{gathered}
D_{\beta}(\lambda) F_{\beta}(\lambda)=P_{\beta_{0}}+\left(P_{\beta_{1}}-P_{\beta_{0}} M_{\beta}\right) \lambda-P_{\beta_{1}} M_{\beta} \lambda^{2} \\
=P_{\beta_{0}}+\left(P_{\beta_{1}}+S_{0}^{+} Q_{1}\right) \lambda=\sum_{h=0}^{1} \lambda^{h} F_{h}(\lambda) .
\end{gathered}
$$

This shows that $\widetilde{F}(\lambda) A(\lambda)=D_{\beta}(\lambda) F_{\beta}(\lambda)$, i.e., see Theorem 2.4 , one has the $\beta$ factorization

$$
A(\lambda)=E_{\beta}(\lambda) D_{\beta}(\lambda) F_{\beta}(\lambda), \quad E_{\beta}(\lambda)=\widetilde{F}(\lambda)^{-1}=\sum_{n=0}^{\infty} E_{\beta, n} \lambda^{n}
$$

where $E_{\beta, 0}=\widetilde{F}(0)^{-1}=\sum_{h=0}^{1} S_{h}$ and the formula for $E_{\beta, n}, n=1,2, \ldots$, is found from $E_{\beta}(\lambda)=A(\lambda) F_{\beta}(\lambda)^{-1} D_{\beta}(\lambda)^{-1}$, see the proof of Theorem 2.4. 


\subsection{Poles of order 2}

Consider the case in which $A(\lambda)^{-1}$ has a pole of order $m=2$ at 0. In this case, (1.5) reads

$$
A(\lambda)^{-1}=\frac{Z_{0}}{\lambda^{2}}+\frac{Z_{1}}{\lambda}+\sum_{n=2}^{\infty} Z_{n} \lambda^{n-2}, \quad \lambda \in D_{\rho} \backslash\{0\}, \quad Z_{0} \neq 0
$$

and (1.8) reads

$$
\begin{aligned}
A_{0} Z_{0} & =0, & Z_{0} A_{0} & =0, \\
A_{0} Z_{1}+A_{1} Z_{0} & =0, & Z_{1} A_{0}+Z_{0} A_{1} & =0, \\
A_{0} Z_{2}+A_{1} Z_{1} & =I, & Z_{2} A_{0}+Z_{1} A_{1} & =I .
\end{aligned}
$$

Recall that $m>1$ implies $\alpha_{1} \subset \alpha_{0}^{\perp}$ and $\beta_{1} \subset \beta_{0}^{\perp}$. Let $a_{2}=\alpha_{0} \oplus \alpha_{1}$ and $b_{2}=\beta_{0} \oplus \beta_{1}$; then one has the orthogonal direct sum decompositions

$$
Y=a_{2} \oplus a_{2}^{\perp}, \quad a_{2}, a_{2}^{\perp} \neq 0, \quad X=b_{2} \oplus b_{2}^{\perp}, \quad b_{2}, b_{2}^{\perp} \neq 0,
$$

and the associated orthogonal projection identities

$$
I=P_{a_{2}}+P_{a_{2}^{\perp}}, \quad I=P_{b_{2}}+P_{b_{2}^{\perp}}, \quad P_{\zeta}, P_{\zeta^{\perp}} \neq 0, \quad \zeta=a_{2}, b_{2} .
$$

Recall that if $m>1$ one has $\operatorname{Im} Z_{0} \subseteq b_{2}^{\perp}$ and $\operatorname{Ker} Z_{0} \supseteq a_{2}$, see (3.4) and (3.5), so that

$$
Z_{0}=P_{b_{2}^{\perp}} Z_{0}, \quad Z_{0}=Z_{0} P_{a_{2}^{\perp}}
$$

Next observe that from the $Y$ and $X$ versions of equation $1, A_{0} Z_{1}+A_{1} Z_{0}=0$ and $Z_{1} A_{0}+Z_{0} A_{1}=0$, one respectively has $A_{0}^{+} A_{0} Z_{1}=-A_{0}^{+} A_{1} Z_{0}$ and $Z_{1} A_{0} A_{0}^{+}=-Z_{0} A_{1} A_{0}^{+}$; hence, see (2.1),

$$
P_{\beta_{0}} Z_{1}=-A_{0}^{+} A_{1} Z_{0}, \quad Z_{1} P_{\alpha_{0}}=-Z_{0} A_{1} A_{0}^{+}
$$

The l.h.s. of the $Y$ version of equation 2 reads $A_{0} Z_{2}+A_{1} Z_{1}=I$; using $P_{\beta_{0}^{\perp}}+$ $P_{\beta_{0}}=I$ one has $A_{1} Z_{1}=A_{1}\left(P_{\beta_{0}^{\perp}}+P_{\beta_{0}}\right) Z_{1}=A_{1} P_{\beta_{0}^{\perp}} Z_{1}+A_{1} P_{\beta_{0}} Z_{1}=A_{1} P_{\beta_{0}^{\perp}} Z_{1}+$ $Q_{2} Z_{0}$, having defined $Q_{2}=-A_{1} A_{0}^{+} A_{1}$. From $P_{\alpha_{0}^{\perp}}+P_{\alpha_{0}}=I$ one finds $A_{1} Z_{1}=\left(P_{\alpha_{0}^{\perp}}+\right.$ $\left.P_{\alpha_{0}}\right) A_{1} P_{\beta_{0}^{\perp}} Z_{1}+Q_{2} Z_{0}=S_{1} Z_{1}+P_{\alpha_{0}} A_{1} P_{\beta_{0}^{\perp}} Z_{1}+Q_{2} Z_{0}$, where $S_{1}=P_{\alpha_{0}^{\perp}} A_{1} P_{\beta_{0}^{\perp}}$ and $\alpha_{1}=\operatorname{Im} S_{1}$. Applying $P_{a_{2}}$ on both sides of $A_{1} Z_{1}=S_{1} Z_{1}+P_{\alpha_{0}} A_{1} P_{\beta_{0}} Z_{1}+Q_{2} Z_{0}$ one has $P_{a_{2}} A_{1} Z_{1}=P_{a_{2}} Q_{2} Z_{0}=S_{2} Z_{0}$, having used $Z_{0}=P_{b_{2}^{\perp}} Z_{0}$ and defined $S_{2}=P_{a_{2}^{\perp}} Q_{2} P_{b_{2}^{\perp}}$, $\alpha_{2}=\operatorname{Im} S_{2}$ and $\beta_{2}=\left(\operatorname{Ker} S_{2}\right)^{\perp}$; note that $\operatorname{Im} S_{2} \subseteq \operatorname{Im} P_{a_{2}^{\perp}}=a_{2}^{\perp}=\left(\alpha_{0} \oplus \alpha_{1}\right)^{\perp}$ and $\operatorname{Ker} S_{2} \supseteq \operatorname{Ker} P_{b_{2}^{\perp}}=\operatorname{Im} P_{b_{2}}=b_{2}=\beta_{0} \oplus \beta_{1}$. This shows that from the 1.h.s. of the $Y$ version of equation $2, A_{0} Z_{2}+A_{1} Z_{1}$, one has 


$$
P_{a_{2}^{\perp}}\left(A_{0} Z_{2}+A_{1} Z_{1}\right)=S_{2} Z_{0}
$$

because $P_{a_{2}} A_{0}=0$ follows from $\operatorname{Im} A_{0}=\alpha_{0}$.

Similarly, starting from the l.h.s. of the $X$ version of equation $2, Z_{2} A_{0}+Z_{1} A_{1}$, and using projections (the details are omitted) one finds

$$
\left(Z_{1} A_{0}+Z_{0} A_{1}\right) P_{b_{2}^{\perp}}=Z_{0} S_{2}
$$

Because the identity is in equations 2, (3.11) and (3.12) respectively imply

$$
S_{2} Z_{0}=P_{a_{2}^{\perp}}, \quad Z_{0} S_{2}=P_{b_{2}^{\perp}}
$$

From $S_{2} Z_{0}=P_{a \frac{\perp}{2}}$ one has

$$
\operatorname{Ker} Z_{0}=\operatorname{Ker} P_{a_{2}^{\perp}}=a_{2}, \quad \operatorname{Im} S_{2}=\operatorname{Im} P_{a_{2}^{\perp}}=a_{2}^{\perp},
$$

and from $Z_{0} S_{2}=P_{b_{2}^{\perp}}$ one finds

$$
\operatorname{Im} Z_{0}=\operatorname{Im} P_{b_{2}^{\perp}}=b_{2}^{\perp}, \quad \operatorname{Ker} S_{2}=\operatorname{Ker} P_{b_{2}^{\perp}}=b_{2} .
$$

This shows that if $m=2$ then

$$
\alpha_{2}=\left(\alpha_{0} \oplus \alpha_{1}\right)^{\perp}, \quad \beta_{2}=\left(\beta_{0} \oplus \beta_{1}\right)^{\perp},
$$

i.e.

$$
X=\beta_{0} \oplus \beta_{1} \oplus \beta_{2}, \quad Y=\alpha_{0} \oplus \alpha_{1} \oplus \alpha_{2}
$$

Conversely, when $m>2$ the identity is not in equations 2 and (3.11) and (3.12) respectively imply

$$
S_{2} Z_{0}=0, \quad Z_{0} S_{2}=0
$$

From $A_{0} Z_{0}=0, S_{1} Z_{0}=0$ and $S_{2} Z_{0}=0$ one has

$$
\operatorname{Im} Z_{0} \subseteq\left(\operatorname{Ker} A_{0} \cap \operatorname{Ker} S_{1} \cap \operatorname{Ker} S_{2}\right)=\beta_{0}^{\perp} \cap \beta_{1}^{\perp} \cap \beta_{2}^{\perp}=\left(\beta_{0} \oplus \beta_{1} \oplus \beta_{2}\right)^{\perp}
$$

and because $\beta_{2}=\left(\beta_{0} \oplus \beta_{1}\right)^{\perp}$ implies $\operatorname{Im} Z_{0}=0$, if $m>2$ then $\beta_{2} \subset\left(\beta_{0} \oplus \beta_{1}\right)^{\perp}$. Thus $\beta_{2}=\left(\beta_{0} \oplus \beta_{1}\right)^{\perp}$ holds if and only if $m=2$.

Similarly, from $Z_{0} A_{0}=0, Z_{0} S_{1}=0$ and $Z_{0} S_{2}=0$ one has

$$
\operatorname{Ker} Z_{0} \supseteq\left(\operatorname{Im} A_{0} \oplus \operatorname{Im} S_{1} \oplus \operatorname{Im} S_{2}\right)=\alpha_{0} \oplus \alpha_{1} \oplus \alpha_{2}
$$

and because $\alpha_{2}=\left(\alpha_{0} \oplus \alpha_{1}\right)^{\perp}$ implies Ker $Z_{0}=Y$ and hence $Z_{0}=0$, if $m>2$ then $\alpha_{2} \subset\left(\alpha_{0} \oplus \alpha_{1}\right)^{\perp}$. Thus $\alpha_{2}=\left(\alpha_{0} \oplus \alpha_{1}\right)^{\perp}$ holds if and only if $m=2$. 
When $m=2$, (3.13) thus reads $S_{2} Z_{0}=P_{\alpha_{2}}$ and $Z_{0} S_{2}=P_{\beta_{2}}$. The former implies $Z_{0} S_{2} Z_{0}=Z_{0} P_{\alpha_{2}}$ and because $Z_{0}=Z_{0} P_{\alpha_{2}}$ one finds $Z_{0} S_{2} Z_{0}=Z_{0}$; similarly, from the latter one has $S_{2} Z_{0} S_{2}=S_{2} P_{\beta_{2}}$ and because $S_{2}=S_{2} P_{\beta_{2}}$ one finds $S_{2} Z_{0} S_{2}=S_{2}$. This shows that, when the pole has order two, $Z_{0}$ is the generalized inverse of $S_{2}$.

This proves the following statement.

Proposition 3.2 (Pole of order two). Let $S_{h}, \alpha_{h}, \beta_{h}, h=0,1$, be as in (3.6) and (3.7) and define

$$
S_{2}=P_{a_{2}^{\perp}} Q_{2} P_{b_{2}^{\perp}}, \quad \alpha_{2}=\operatorname{Im} S_{2}, \quad \beta_{2}=\left(\operatorname{Ker} S_{2}\right)^{\perp},
$$

where

$$
a_{2}=\alpha_{0} \oplus \alpha_{1}, \quad b_{2}=\beta_{0} \oplus \beta_{1}, \quad Q_{2}=-Q_{1} S_{0}^{+} Q_{1}, \quad Q_{1}=A_{1} .
$$

The following statements are equivalent:

(i) $A(\lambda)^{-1}$ has a pole of order $m=2$ at 0 ,

(ii) $X=\beta_{0} \oplus \beta_{1} \oplus \beta_{2}$, where $\beta_{h}$ is closed and $\beta_{0}, \beta_{2} \neq 0$,

(iii) $Y=\alpha_{0} \oplus \alpha_{1} \oplus \alpha_{2}$, where $\alpha_{h}$ is closed and $\alpha_{0}, \alpha_{2} \neq 0$,

(iv) $Z_{0}=S_{2}^{+}$.

Thus, when the pole has order two, $Z_{0}$ is such that

$$
\operatorname{Im} Z_{0}=\beta_{2}, \quad \operatorname{Ker} Z_{0}=\alpha_{0} \oplus \alpha_{1}
$$

Observe that these quantities are all expressed in terms of $A_{0}$ and $A_{1}$ via the definitions in (3.6), (3.7) and (3.14); further note that Proposition 3.2 is found by setting $m=2$ in Theorem 2.3.

Next we illustrate the derivation of the $\beta$ factorization $A(\lambda)=E_{\beta}(\lambda) D_{\beta}(\lambda) F_{\beta}(\lambda)$ in Theorem 2.4 for $m=2$ (the $\alpha$ factorization is obtained similarly from the $X$ versions of the fundamental equations). From the above one has

$$
P_{\beta_{0}} Z_{0}=0, \quad P_{\beta_{1}} Z_{0}=0, \quad P_{\beta_{2}} Z_{0}=S_{2}^{+},
$$

from $A_{0} Z_{1}+A_{1} Z_{0}=0$ it follows that $A_{0}^{+} A_{0} Z_{1}+A_{0}^{+} A_{1} Z_{0}=0$ and hence, see (2.1),

$$
P_{\beta_{0}} Z_{1}=-S_{0}^{+} Q_{1} Z_{0}, \quad S_{0}=A_{0}, \quad Q_{1}=A_{1},
$$

and from $A_{0} Z_{2}+A_{1} Z_{1}=I$ one has $A_{0}^{+} A_{0} Z_{2}+A_{0}^{+} A_{1} Z_{1}=A_{0}^{+}$, i.e.

$$
P_{\beta_{0}} Z_{2}=S_{0}^{+}-S_{0}^{+} Q_{1} Z_{1}
$$

Moreover, because $S_{1}^{+} \alpha_{0}=0, S_{1}^{+}\left(A_{0} Z_{2}+A_{1} Z_{1}\right)=S_{1}^{+} A_{1} Z_{1}$ and from $A_{0} Z_{2}+A_{1} Z_{1}=I$ one has $S_{1}^{+} A_{1} Z_{1}=S_{1}^{+}$; because, see above, $A_{1} Z_{1}=S_{1} Z_{1}+P_{\alpha_{0}} A_{1} P_{\beta_{0}^{\perp}} Z_{1}+Q_{2} Z_{0}$, one has $S_{1}^{+} A_{1} Z_{1}=S_{1}^{+} S_{1} Z_{1}+S_{1}^{+} P_{\alpha_{0}} A_{1} P_{\beta_{0}} Z_{1}+S_{1}^{+} Q_{2} Z_{0}=P_{\beta_{1}} Z_{1}+S_{1}^{+} Q_{2} Z_{0}$, i.e. 


$$
P_{\beta_{1}} Z_{1}=S_{1}^{+}-S_{1}^{+} Q_{2} Z_{0}
$$

Thus

$$
\begin{gathered}
P_{\beta_{0}} A(\lambda)^{-1}=\frac{P_{\beta_{0}} Z_{0}}{\lambda^{2}}+\frac{P_{\beta_{0}} Z_{1}}{\lambda}+P_{\beta_{0}} Z_{2}+\sum_{n=3}^{\infty} P_{\beta_{0}} Z_{n} \lambda^{n-2} \\
=-\frac{S_{0}^{+} Q_{1} Z_{0}}{\lambda}+\left(S_{0}^{+}-S_{0}^{+} Q_{1} Z_{1}\right)+\sum_{n=1}^{\infty} P_{\beta_{0}} Z_{n+2} \lambda^{n} .
\end{gathered}
$$

Applying $\lambda S_{0}^{+} Q_{1}$ to $A(\lambda)^{-1}$ one has

$$
\left(\lambda S_{0}^{+} Q_{1}\right) A(\lambda)^{-1}=\frac{S_{0}^{+} Q_{1} Z_{0}}{\lambda}+S_{0}^{+} Q_{1} Z_{1}+\sum_{n=1}^{\infty} S_{0}^{+} Q_{1} Z_{n+1} \lambda^{n}
$$

and summing up the two expressions one has

$$
\left(P_{\beta_{0}}+\lambda S_{0}^{+} A_{1}\right) A(\lambda)^{-1}=S_{0}^{+}+\sum_{n=1}^{\infty}\left(P_{\beta_{0}} Z_{n+2}+S_{0}^{+} Q_{1} Z_{n+1}\right) \lambda^{n}
$$

i.e., see Proposition 4.2 below,

$$
F_{0}(\lambda) A(\lambda)^{-1}=\widetilde{F}_{0}(\lambda)
$$

where $F_{0}(\lambda)=P_{\beta_{0}}+\lambda S_{0}^{+} A_{1}, \widetilde{F}_{0}(\lambda)=S_{0}^{+}+\sum_{n=1}^{\infty}\left(P_{\beta_{0}} Z_{n+2}+S_{0}^{+} Q_{1} Z_{n+1}\right) \lambda^{n}$ is analytic on $D_{\rho}$ and $\widetilde{F}_{0}(0)=S_{0}^{+} \neq 0$. Moreover,

$$
\begin{gathered}
P_{\beta_{1}} A(\lambda)^{-1}=\frac{P_{\beta_{1}} Z_{0}}{\lambda^{2}}+\frac{P_{\beta_{1}} Z_{1}}{\lambda}+\sum_{n=2}^{\infty} P_{\beta_{1}} Z_{n} \lambda^{n-2} \\
=\frac{S_{1}^{+}-S_{1}^{+} Q_{2} Z_{0}}{\lambda}+\sum_{n=0}^{\infty} P_{\beta_{1}} Z_{n+2} \lambda^{n}
\end{gathered}
$$

Applying $\lambda S_{1}^{+} Q_{2}$ to $A(\lambda)^{-1}$ one has

$$
\left(\lambda S_{1}^{+} Q_{2}\right) A(\lambda)^{-1}=\frac{S_{1}^{+} Q_{2} Z_{0}}{\lambda}+\sum_{n=0}^{\infty} S_{1}^{+} Q_{2} Z_{n+1} \lambda^{n}
$$

and summing up the two expressions one has

$$
\left(P_{\beta_{1}}+\lambda S_{1}^{+} Q_{2}\right) A(\lambda)^{-1}=\frac{S_{1}^{+}}{\lambda}+\sum_{n=0}^{\infty}\left(P_{\beta_{1}} Z_{n+2}+S_{1}^{+} Q_{2} Z_{n+1}\right) \lambda^{n},
$$

i.e., see Proposition 4.2 below, 


$$
F_{1}(\lambda) A(\lambda)^{-1}=\lambda^{-1} \widetilde{F}_{1}(\lambda)
$$

where $F_{0}(\lambda)=P_{\beta_{1}}+\lambda S_{1}^{+} Q_{2}, \widetilde{F}_{1}(\lambda)=S_{1}^{+}+\sum_{n=0}^{\infty}\left(P_{\beta_{1}} Z_{n+2}+S_{1}^{+} Q_{2} Z_{n+1}\right) \lambda^{n+1}$ is analytic on $D_{\rho}$ and $\widetilde{F}_{1}(0)=S_{1}^{+} \neq 0$. Finally,

$$
P_{\beta_{2}} A(\lambda)^{-1}=\frac{P_{\beta_{2}} Z_{0}}{\lambda^{2}}+\sum_{n=1}^{\infty} P_{\beta_{2}} Z_{n} \lambda^{n-2}=\frac{S_{2}^{+}}{\lambda^{2}}+\sum_{n=1}^{\infty} P_{\beta_{2}} Z_{n} \lambda^{n-2}
$$

i.e., see Proposition 4.2 below,

$$
F_{2}(\lambda) A(\lambda)^{-1}=\lambda^{-2} \widetilde{F}_{2}(\lambda)
$$

where $F_{2}(\lambda)=P_{\beta_{2}}, \widetilde{F}_{2}(\lambda)=S_{2}^{+}+\sum_{n=1}^{\infty} P_{\beta_{2}} Z_{n} \lambda^{n}$ is analytic on $D_{\rho}$ and $\widetilde{F}_{2}(0)=S_{2}^{+} \neq 0$. Hence one has

$$
\widetilde{F}_{h}(\lambda) A(\lambda)=\lambda^{h} F_{h}(\lambda), \quad \widetilde{F}_{0}(\lambda)=S_{h}^{+}, \quad h=0,1,2,
$$

where $\operatorname{Im} S_{h}^{+}=\beta_{h}$ and $\operatorname{Ker} S_{h}^{+}=\alpha_{h}^{\perp}$, see (2.1). Summing over $h$ one finds

$$
\widetilde{F}(\lambda) A(\lambda)=\sum_{h=0}^{2} \lambda^{h} F_{h}(\lambda), \quad \widetilde{F}(\lambda)=\sum_{h=0}^{2} \widetilde{F}_{h}(\lambda),
$$

where $\widetilde{F}(\lambda)$ is analytic on $D_{\rho}, \widetilde{F}(0)=\sum_{h=0}^{2} S_{h}^{+}$is invertible and

$$
\sum_{h=0}^{2} \lambda^{h} F_{h}(\lambda)=P_{\beta_{0}}+\lambda\left(P_{\beta_{1}}+S_{0}^{+} Q_{1}\right)+\lambda^{2}\left(P_{\beta_{2}}+S_{1}^{+} Q_{2}\right)
$$

On the other hand, letting

$$
D_{\beta}(\lambda)=P_{\beta_{0}}+P_{\beta_{1}} \lambda+P_{\beta_{2}} \lambda^{2}, \quad F_{\beta}(\lambda)=I-M_{\beta} \lambda, \quad M_{\beta}=-\sum_{h=0}^{1} S_{h}^{+} Q_{h+1},
$$

one has $P_{\beta_{h}} M_{\beta}=-P_{\beta_{h}} S_{h}^{+} Q_{h+1}=-S_{h}^{+} Q_{h+1}, h=0,1$, and $P_{\beta_{2}} M_{\beta}=0$ and hence

$$
\begin{gathered}
D_{\beta}(\lambda) F_{\beta}(\lambda)=P_{\beta_{0}}+\left(P_{\beta_{1}}-P_{\beta_{0}} M_{\beta}\right) \lambda+\left(P_{\beta_{2}}-P_{\beta_{1}} M_{\beta}\right) \lambda^{2}-P_{\beta_{2}} M_{\beta} \lambda^{3} \\
=P_{\beta_{0}}+\left(P_{\beta_{1}}+S_{0}^{+} Q_{1}\right) \lambda+\left(P_{\beta_{1}}+S_{1}^{+} Q_{2}\right) \lambda^{2}=\sum_{h=0}^{2} \lambda^{h} F_{h}(\lambda) .
\end{gathered}
$$

This shows that $\widetilde{F}(\lambda) A(\lambda)=D_{\beta}(\lambda) F_{\beta}(\lambda)$, i.e., see Theorem 2.4 , one has the $\beta$ factorization

$$
A(\lambda)=E_{\beta}(\lambda) D_{\beta}(\lambda) F_{\beta}(\lambda), \quad E_{\beta}(\lambda)=\widetilde{F}(\lambda)^{-1}=\sum_{n=0}^{\infty} E_{\beta, n} \lambda^{n}
$$


where $E_{\beta, 0}=\widetilde{F}(0)^{-1}=\sum_{h=0}^{2} S_{h}$ and the formula for $E_{\beta, n}, n=1,2, \ldots$, is found from $E_{\beta}(\lambda)=A(\lambda) F_{\beta}(\lambda)^{-1} D_{\beta}(\lambda)^{-1}$, see the proof of Theorem 2.4.

As the results in the next section show, the same structure applies to poles of order $m \in$ $\mathbb{N}$. The key result is presented in Lemma 4.1: this provides the subspace decompositions of the fundamental equations that describe the underlying structure.

\section{Order of the pole and factorizations}

This section reports results in Lemma 4.1, which is of central relevance in the analysis and provides the subspace decompositions of the fundamental equations in (1.8), and in Theorem 2.3, which characterizes the order of the pole in terms of the orthogonal direct sum decompositions $X=\bigoplus_{h=0}^{m} \beta_{h}$ and $Y=\bigoplus_{h=0}^{m} \alpha_{h}$. A construction of root functions of $A(\lambda)^{-1}$ at 0 is provided in Proposition 4.2 and this leads to two alternative constructions in Theorem 2.4 of the factorization $A(\lambda)=E(\lambda) D(\lambda) F(\lambda)$, where $E(\lambda), F(\lambda)$ are analytic and invertible on $D_{\rho}$ and $D(\lambda)$ describes the singularity of $A(\lambda)$ at 0 . This in turn allows to provide formulas for the coefficients of the resolvent in Corollary 4.3.

The next statement reports consequences of system (1.8) that are derived using the projection identities that correspond to the subspaces defined recursively by the local subspace decomposition. This lemma motivates Definition 2.2 and plays a central role in the analysis, as all the results in the paper are consequences of (4.1) and (4.2) below.

Lemma 4.1 (Subspace decompositions of (1.8)). Consider Definition 2.2 and let $P_{a_{0}^{\perp}}=$ $P_{b_{0}^{\perp}}=I$ and $Z_{-1}=0$; then the $Y$ version of equation $n+h \leq m$ in system (1.8) implies

$$
S_{h} Z_{n}+P_{a_{h}} Q_{h+1} Z_{n-1}=1_{n+h=m} P_{a_{h}^{\perp}}, \quad h=0, \ldots, m-n .
$$

Similarly, the $X$ version of equation $n+h \leq m$ in system (1.8) implies

$$
Z_{n} S_{h}+Z_{n-1} Q_{h+1} P_{b_{h}^{\perp}}=1_{n+h=m} P_{b_{h}^{\perp}}, \quad h=0, \ldots, m-n .
$$

First note that for $h=0,(4.1)$ and (4.2) coincide with (1.8); in fact, because $P_{a_{0}^{\perp}}=I$, $P_{b_{0}^{\perp}}=I, S_{0}=A_{0}, Q_{1}=A_{1}$, and $Z_{-1}=0$, one has

$$
A_{0} Z_{n}+A_{1} Z_{n-1}=1_{n=m} I, \quad Z_{n} A_{0}+Z_{n-1} A_{1}=1_{n=m} I, \quad n=0, \ldots, m .
$$

More informative relations arise from (4.1) and (4.2) by setting, for example, $n=0$ and $h=m=1,2$; for instance, (4.1) respectively delivers (3.3) and (3.13), which led to prove Propositions 3.1, 3.2. The same structure is found for poles of any order, i.e. setting $n=0$ and $h=m$ in (4.1) and (4.2), one has

$$
S_{m} Z_{0}=P_{a_{m}^{\perp}}, \quad Z_{0} S_{m}=P_{b_{m}^{\perp}}
$$

This leads to the statement in Theorem 2.3, see the proof in Appendix A; note that from $(i v)$ in Theorem 2.3 one has 


$$
\operatorname{Im} Z_{0}=\beta_{m}, \quad \operatorname{Ker} Z_{0}=\alpha_{m}^{\perp}
$$

Further observe that (4.1) and (4.2) show how to linearly combine $Z_{n}$ and $Z_{n-1}$ in order to get the zero operator; that is, they provide a construction of the root functions of $A(\lambda)^{-1}$ at 0 . In fact, applying $S_{h}^{+}$to (4.1) and (4.2) to $S_{h}^{+}$and using (2.1) one has

$$
\begin{array}{ll}
P_{\beta_{h}} Z_{n}+S_{h}^{+} Q_{h+1} Z_{n-1}=1_{n+h=m} S_{h}^{+}, & h=0, \ldots, m-n, \\
Z_{n} P_{\alpha_{h}}+Z_{n-1} Q_{h+1} S_{h}^{+}=1_{n+h=m} S_{h}^{+}, & h=0, \ldots, m-n .
\end{array}
$$

Note that by setting $m=1$ and $n=0,1$ in (4.3), one has

$$
P_{\beta_{0}} Z_{0}=0, \quad P_{\beta_{1}} Z_{0}=S_{1}^{+}, \quad P_{\beta_{0}} Z_{1}=S_{0}^{+}-S_{0}^{+} Q_{1} Z_{0},
$$

i.e. (3.8) and (3.9), which led to prove the $\beta$ factorization when $m=1$. Similarly, setting $m=2$ and $n=0,1,2$ in (4.3), one has

$$
\begin{gathered}
P_{\beta_{0}} Z_{0}=0, \quad P_{\beta_{1}} Z_{0}=0, \quad P_{\beta_{2}} Z_{0}=S_{2}^{+}, \\
P_{\beta_{0}} Z_{1}=-S_{0}^{+} Q_{1} Z_{0}, \quad P_{\beta_{1}} Z_{1}=S_{1}^{+}-S_{1}^{+} Q_{2} Z_{0}, \\
P_{\beta_{0}} Z_{2}=S_{0}^{+}-S_{0}^{+} Q_{1} Z_{1},
\end{gathered}
$$

i.e. (3.15), (3.16), (3.17), and (3.18), which led to prove the $\beta$ factorization when $m=2$.

The same structure is found for poles of any order and leads to the following statement.

Proposition 4.2 (Root functions). Consider Definition 2.2 and for $h=0, \ldots, m$, let

$$
F_{h}(\lambda)=P_{\beta_{h}}+\lambda 1_{h \neq m} S_{h}^{+} Q_{h+1}, \quad E_{h}(\lambda)=P_{\alpha_{h}}+\lambda 1_{h \neq m} Q_{h+1} S_{h}^{+}
$$

Then for $h=0, \ldots, m$, one has

$$
F_{h}(\lambda) A(\lambda)^{-1}=\lambda^{-h} \widetilde{F}_{h}(\lambda), \quad A(\lambda)^{-1} E_{h}(\lambda)=\lambda^{-h} \widetilde{E}_{h}(\lambda)
$$

where $\widetilde{F}_{h}(\lambda)$ and $\widetilde{E}_{h}(\lambda)$ are analytic on $D_{\rho}$ and $\widetilde{F}_{h}(0)=\widetilde{E}_{h}(0)=S_{h}^{+} \neq 0$.

This shows that $F_{h}(\lambda) \in \mathscr{B}_{X}$ and $E_{h}(\lambda) \in \mathscr{B}_{Y}$ are root functions of order $-h$ of $A(\lambda)^{-1}$ at 0 . In fact, $F_{h}(\lambda)$ and $E_{h}(\lambda)$ factor out $\lambda^{m-h}$ from $Z(\lambda)=\sum_{n=0}^{\infty} Z_{n} \lambda^{n}$ in $A(\lambda)^{-1}=\lambda^{-m} Z(\lambda)$ hence decreasing the order of the pole in $F_{h}(\lambda) A(\lambda)^{-1}$ and $A(\lambda)^{-1} E_{h}(\lambda)$ to $h=0, \ldots, m-1$.

This leads to the statement in Theorem 2.4, see the proof in Appendix A. Theorem 2.4 provides two alternative constructions of the factorization $A(\lambda)=E(\lambda) D(\lambda) F(\lambda)$, where $E(\lambda), F(\lambda)$ are analytic and invertible on $D_{\rho}$ and $D(\lambda)$ describes the singularity of $A(\lambda)$ at 0 . The existence of the factorization $A(\lambda)=E(\lambda) D(\lambda) F(\lambda)$ for an operator function with values in the algebra of all bounded linear operators acting in a Banach space which 
are holomorphic in some neighbourhood of some point, except possibly at this point itself, is discussed in Gohberg and Sigal [17] in the special case of $A_{0}$ being Fredholm of index 0, i.e. $\operatorname{dim} \operatorname{Ker} A_{0}$ and $\operatorname{codim} \operatorname{Im} A_{0}=\operatorname{dim}\left(Y / \operatorname{Im} A_{0}\right)$ are finite and equal. The same kind of factorization holds in the present setup and Theorem 2.4 provides two alternative constructions of its factors.

Note that from $E_{\beta, 0}=F_{\alpha, 0}=\sum_{h=0}^{m} S_{h}$ one has $E_{\beta, 0}^{-1}=F_{\alpha, 0}^{-1}=\sum_{h=0}^{m} S_{h}^{+}$; further observe that because $S_{h} S_{h}^{+}=P_{\alpha_{h}}$ and $S_{h}^{+} S_{h}=P_{\beta_{h}}$ one has $S_{h} P_{\beta_{h}} S_{h}^{+}=P_{\alpha_{h}}$ and $S_{h}^{+} P_{\alpha_{h}} S_{h}=P_{\beta_{h}} ;$ hence

$$
E_{\beta, 0}^{-1} D_{\alpha}(\lambda) E_{\beta, 0}=\left(\sum_{h=0}^{m} S_{h}^{+}\right) \sum_{h=0}^{m} P_{\alpha_{h}} \lambda^{h}\left(\sum_{h=0}^{m} S_{h}\right)=\sum_{h=0}^{m}\left(S_{h}^{+} P_{\alpha_{h}} S_{h}\right) \lambda^{h}=D_{\beta}(\lambda)
$$

and thus

$$
A(\lambda)=E_{\beta}(\lambda) D_{\beta}(\lambda) F_{\beta}(\lambda)=\widetilde{E}_{\beta}(\lambda) D_{\alpha}(\lambda) \widetilde{F}_{\beta}(\lambda)
$$

where $\widetilde{E}_{\beta}(0)=E_{\beta}(0) E_{\beta, 0}^{-1}=I$ and $\widetilde{F}_{\beta}(0)=E_{\beta, 0} F_{\beta}(0)=\sum_{h=0}^{m} S_{h}$. This illustrates that the factors in (2.2) are not unique.

Finally note that from (2.2) one has

$$
A(\lambda)^{-1}=F_{\zeta}(\lambda)^{-1} D_{\zeta}(\lambda)^{-1} E_{\zeta}(\lambda)^{-1}=\sum_{n=0}^{\infty} Z_{n} \lambda^{n-m}, \quad \zeta=\alpha, \beta
$$

hence the following statement.

Corollary 4.3 (A formula for $Z_{n}, n=0,1, \ldots$ ). For $n=0,1, \ldots, Z_{n}$ can be computed as

$$
Z_{n}=\sum_{k=0}^{n} B_{\beta, k} C_{\beta, n-k}=\sum_{k=0}^{n} B_{\alpha, n-k} C_{\alpha, k}
$$

where

$$
\begin{gathered}
B_{\beta, n}=\left\{\begin{array}{c}
\sum_{k=0}^{n} M_{\beta}^{k} P_{\beta_{m-n+k}}, \\
M_{\beta}^{n-m} B_{\beta, m}
\end{array}\right. \\
C_{\alpha, n}=\left\{\begin{array}{cc}
\sum_{k=0}^{n} P_{\alpha_{m-n+k}}\left(M_{\alpha}^{*}\right)^{k}, & n=0, \ldots, m \\
C_{\alpha, m}\left(M_{\alpha}^{*}\right)^{n-m}, & n=m+1, m+2, \ldots
\end{array},\right. \\
C_{\beta, n}=-\sum_{k=1}^{n} E_{\beta, 0}^{-1} E_{\beta, k} C_{\beta, n-k} \\
B_{\alpha, n}=-\sum_{k=1}^{n} F_{\alpha, 0}^{-1} F_{\alpha, k} B_{\alpha, n-k}
\end{gathered}
$$

and

$$
C_{\beta, 0}=B_{\alpha, 0}=E_{\beta, 0}^{-1}=F_{\alpha, 0}^{-1}=\sum_{h=0}^{m} S_{h}^{+}, \quad \begin{aligned}
& E_{\beta, k}=P_{\alpha_{0}^{\perp}} A_{1} M_{\beta}^{k-1} B_{\beta, m} \\
& F_{\alpha, k}=C_{\alpha, m}\left(M_{\alpha}^{*}\right)^{k-1} A_{1} P_{\beta_{0}^{\perp}}
\end{aligned} .
$$




\section{Chains and subspaces}

This section presents the following results: Proposition 5.1 shows that $M_{\beta}$ and $M_{\alpha}$ deliver chains for $A(\lambda)$ and $A(\lambda)^{*}$ at 0 and Theorem 2.5 links these chains to the subspaces in the [10] direct sum decompositions. Theorem 2.6 characterizes the image and the null space of the operators in the principal part of the resolvent via the same quantities and Corollary 5.2 provides recursions for $\operatorname{Im} Z_{n}$ and $\operatorname{Ker} Z_{n}, n=0, \ldots, m-1$.

From (2.2) one has $A(\lambda) F_{\beta}(\lambda)^{-1}=E_{\beta}(\lambda) D_{\beta}(\lambda)$ and $E_{\alpha}(\lambda)^{-1} A(\lambda)=D_{\alpha}(\lambda) F_{\alpha}(\lambda)$; direct manipulations of these equations allow to show that the operators $M_{\beta}$ and $M_{\alpha}$ introduced in Definition 2.2 deliver chains for $A(\lambda)$ and $A(\lambda)^{*}$ at 0 .

Proposition 5.1 (Chains). Consider Definition 2.2 and for $h=1, \ldots, m$, let $x_{h, 0} \in \beta_{h}$ and $y_{h, 0} \in \alpha_{h}$ be nonzero vectors and further define

$$
x_{h, n}=M_{\beta} x_{h, n-1}, \quad y_{h, n}=M_{\alpha} y_{h, n-1}, \quad n=1,2, \ldots
$$

Then

$$
\begin{array}{lll}
A_{0} x_{h, 0}=0, & A_{0} x_{h, n}+A_{1} x_{h, n-1}=0, & n=1, \ldots, h-1, \\
A_{0}^{*} y_{h, 0}=0, & A_{0}^{*} y_{h, n}+A_{1}^{*} y_{h, n-1}=0, & n=1, \ldots, h-1,
\end{array}
$$

and

$$
A_{0} x_{h, h}+A_{1} x_{h, h-1} \neq 0, \quad A_{0}^{*} y_{h, h}+A_{1}^{*} y_{h, h-1} \neq 0 .
$$

Moreover, the vectors in the $X$ and $Y$-chains

$$
x_{h, 0}, \ldots, x_{h, h-1}, \quad y_{h, 0}, \ldots, y_{h, h-1}, \quad h=1, \ldots, m,
$$

are linearly independent.

This shows that $x_{h, 0}, x_{h, 1}, \ldots, x_{h, h-1}$ is a chain in $X$ of maximal length $h=1, \ldots, m$ for $A(\lambda)=A_{0}+A_{1} \lambda$ at 0 and similarly, $y_{h, 0}, y_{h, 1}, \ldots, y_{h, h-1}$ is a chain in $Y$ of maximal length $h=1, \ldots, m$ for $A(\lambda)^{*}=A_{0}^{*}+A_{1}^{*} \lambda$ at 0 . Remark that, because Ker $A_{0}=\beta_{0}^{\perp}$, no $X$-chain is associated to the subspace $\beta_{0}$ and similarly, because $\operatorname{Ker} A_{0}^{*}=\left(\operatorname{Im} A_{0}\right)^{\perp}=$ $\alpha_{0}^{\perp}$, no $Y$-chain is associated to the subspace $\alpha_{0}$; moreover, the orthogonal direct sum decompositions $\operatorname{Ker} A_{0}=\bigoplus_{h=1}^{m} \beta_{h}$ and $\operatorname{Ker} A_{0}^{*}=\left(\operatorname{Im} A_{0}\right)^{\perp}=\bigoplus_{h=1}^{m} \alpha_{h}$ isolate the subspaces that deliver chains that have the same maximal length.

Consider the $X$-chains (the interpretation of the $Y$-chain is similar) and the orthogonal direct sum decomposition Ker $A_{0}=\bigoplus_{h=1}^{m} \beta_{h}$. Fix a nonzero vector $x_{h, 0} \in \beta_{h}$ and repeatedly apply the $M_{\beta}$ operator to define $x_{h, n}=M_{\beta}^{n} x_{h, 0}, n=1,2, \ldots$ Proposition 5.1 shows that such a chain ends after $h-1$ repetitions, i.e. the chain associated to $x_{h, 0} \in \beta_{h}$ has maximal length $h$. Next take another nonzero vector $\widetilde{x}_{h, 0} \in \beta_{h}$ which is 
linearly independent of $x_{h, 0}$ and construct in the same way the chain of maximal length $h$ associated to $\widetilde{x}_{h, 0} \in \beta_{h}$. Proposition 5.1 shows that the vectors in these two chains are linearly independent. This process is repeated as long as there are vectors in $\beta_{h}$ from which one can start a chain. Because of linear independence, the subspace defined by the $X$-chains of maximal length $h$ associated to the subspace $\beta_{h}$ is thus $\bigoplus_{n=0}^{h-1} M_{\beta}^{n} \beta_{h}$. Collecting together the subspaces associated to the different $\beta_{h}$, one has that the subspace associated to $\operatorname{Ker} A_{0}=\bigoplus_{h=1}^{m} \beta_{h}$ is

$$
\bigoplus_{h=1}^{m} \bigoplus_{n=0}^{h-1} M_{\beta}^{n} \beta_{h}
$$

Similarly, the subspace defined by the $Y$-chains of maximal length $h$ associated to the subspace $\alpha_{h}$ is $\bigoplus_{n=0}^{h-1} M_{\alpha}^{n} \alpha_{h}$ and the one associated to $\operatorname{Ker} A_{0}^{*}=\left(\operatorname{Im} A_{0}\right)^{\perp}=\bigoplus_{h=1}^{m} \alpha_{h}$ is

$$
\bigoplus_{h=1}^{m} \bigoplus_{n=0}^{h-1} M_{\alpha}^{n} \alpha_{h}
$$

This leads to the statement in Theorem 2.5, see the proof in Appendix A. Theorem 2.5 links the present results with the ones in Bart and Lay [10], showing how the subspaces in (1.6) can be constructed using the quantities defined by the local subspace decomposition. This allows to construct the operators $Z_{n \mathscr{N}^{\prime}}, Z_{n \mathscr{R}^{\prime}}$ in (1.7) and the projections $P_{\mathscr{N}, \mathscr{R}}$, $P_{\mathscr{R}, \mathscr{N}}, P_{\mathscr{N}^{\prime}, \mathscr{R}^{\prime}}, P_{\mathscr{R}^{\prime}, N^{\prime}}$ associated to the direct sum decompositions in (1.6). It thus provides a way to compute the resolvent and the logarithmic residues in terms of the quantities defined by the local subspace decomposition.

The linear independence of the vectors in the $X$ and $Y$-chains leads to the statement in Theorem 2.6, see the proof in Appendix A. Note that for $n=0$, Theorem 2.6 gives

$$
\operatorname{Im} Z_{0}=\beta_{m}, \quad \operatorname{Ker} Z_{0}=\alpha_{m}^{\perp}
$$

which are already known from Theorem 2.3, and for $n=m-1$,

$$
\operatorname{Im} Z_{m-1}=\bigoplus_{h=1}^{m} \bigoplus_{k=0}^{h-1} M_{\beta}^{k} \beta_{h}=\mathscr{N}_{m}, \quad \operatorname{Ker} Z_{m-1}=\left(\bigoplus_{h=1}^{m} \bigoplus_{k=0}^{h-1} M_{\alpha}^{k} \alpha_{h}\right)^{\perp}=\mathscr{R}_{m}^{\prime}
$$

which are already known from Theorem 2.5 since $P_{\mathscr{N}, \mathscr{R}}=Z_{m-1} A_{1}, P_{\mathscr{N}^{\prime}, \mathscr{R}^{\prime}}=A_{1} Z_{m-1}$ and $\operatorname{Im} P_{\mathscr{N}, \mathscr{R}}=\operatorname{Im} Z_{m-1}, \operatorname{Ker} P_{\mathscr{N}^{\prime}, \mathscr{R}^{\prime}}=\operatorname{Ker} Z_{m-1}$.

Further note that for $n=1, \ldots, m-2$, one has

$$
\operatorname{Im} Z_{1}=\beta_{m-1} \oplus \beta_{m} \oplus M_{\beta} \beta_{m}, \quad \operatorname{Ker} Z_{1}=\left(\alpha_{m-1} \oplus \alpha_{m} \oplus M_{\alpha} \alpha_{m}\right)^{\perp}
$$




$$
\operatorname{Im} Z_{m-2}=\bigoplus_{h=2}^{m} \bigoplus_{k=0}^{h-(m-2)+n} M_{\beta}^{k} \beta_{h}, \quad \operatorname{Ker} Z_{n}=\left(\bigoplus_{h=2}^{m} \bigoplus_{k=0}^{h-(m-2)+n} M_{\alpha}^{k} \alpha_{h}\right)^{\perp}
$$

This shows in which way $\operatorname{Im} Z_{n}$ and $\operatorname{Ker} Z_{n}, n=0, \ldots, m-1$, respectively form an increasing and a decreasing sequence of nested subspaces, see the next proposition.

Corollary 5.2 (Recursions for $\operatorname{Im} Z_{n}$ and $\operatorname{Ker} Z_{n}, n=0, \ldots, m-1$ ). Let

$$
\xi_{\beta, n}=\bigoplus_{k=0}^{n} M_{\beta}^{k} \beta_{m-n+k}, \quad \xi_{\alpha, n}=\bigoplus_{k=0}^{n} M_{\alpha}^{k} \alpha_{m-n+k}, \quad n=1, \ldots, m-1
$$

Then $\operatorname{Im} Z_{0}=\beta_{m}$, Ker $Z_{0}=\alpha_{m}^{\perp}$ and

$$
\operatorname{Im} Z_{n}=\operatorname{Im} Z_{n-1} \oplus \xi_{\beta, n}, \quad \operatorname{Ker} Z_{n}=\operatorname{ker} Z_{n-1} \cap \xi_{\alpha, n}^{\perp}, \quad n=1, \ldots, m-1
$$

Remark that, because $0 \neq M_{\beta}^{n} \beta_{m} \subseteq \xi_{\beta, n}$ and $0 \neq M_{\alpha}^{n} \alpha_{m} \subseteq \xi_{\beta, n}$, one has the strict inclusions

$$
\operatorname{Im} Z_{n} \supset \operatorname{Im} Z_{n-1}, \quad \operatorname{Ker} Z_{n} \subset \operatorname{Ker} Z_{n-1}, \quad n=1, \ldots, m-1 .
$$

\section{Special case: $A_{0}$ is Fredholm of index 0}

This sections considers the special case of $A_{0}$ being Fredholm of index 0, i.e. $\operatorname{dim} \operatorname{Ker} A_{0}$ and $\operatorname{codim} \operatorname{Im} A_{0}=\operatorname{dim}\left(Y / \operatorname{Im} A_{0}\right)$ are finite and equal.

It is well known, see e.g. Chapter XI in [15], that if $A_{0}$ is Fredholm of index 0 then $Z_{n}, n=0, \ldots, m-1$, has finite rank and $Z_{m}$ is Fredholm of index 0. Moreover, in the Gohberg and Sigal [17] factorization $A(\lambda)=E(\lambda) D(\lambda) F(\lambda)$ one can choose

$$
D(\lambda)=D_{0}+D_{1} \lambda^{\kappa_{1}}+\cdots+D_{s} \lambda^{\kappa_{s}},
$$

where $0<\kappa_{1} \leq \kappa_{2} \leq \cdots \leq \kappa_{s}<\infty$ are positive integers, $D_{0}$ is Fredholm of index $0, D_{1}, \ldots, D_{s}$ have rank one, and $D_{0}, D_{1}, \ldots, D_{s}$ are mutually disjoint projections that decompose the identity.

$D(\lambda)$ is the local Smith form of $A(\lambda)$ at 0 and $E(\lambda), F(\lambda)$ are extended canonical systems of root functions. The integers $0<\kappa_{1} \leq \kappa_{2} \leq \cdots \leq \kappa_{s}<\infty$ are the partial multiplicities of $A(\lambda)$ at 0 ; remark that they are uniquely defined and not necessarily distinct. Their sum $\sum_{n=1}^{s} \kappa_{n}<\infty$ is the algebraic multiplicity of $A(\lambda)$ at 0 and it is denoted by $\kappa(A)$.

The operator version of the logarithmic residue theorem, see [17], states that

$$
\kappa(A)=\operatorname{trace}\left(\frac{1}{2 \pi i} \int_{\partial D_{\rho}} A(\lambda)^{-1} A_{1} d \lambda\right)=\operatorname{trace}\left(\frac{1}{2 \pi i} \int_{\partial D_{\rho}} A_{1} A(\lambda)^{-1} d \lambda\right) .
$$


Because $P_{\mathscr{N}, \mathscr{R}}=\frac{1}{2 \pi i} \int_{\partial D_{\rho}} A(\lambda)^{-1} A_{1} d \lambda$ and $P_{\mathscr{N}^{\prime}, \mathscr{R}^{\prime}}=\frac{1}{2 \pi i} \int_{\partial D_{\rho}} A_{1} A(\lambda)^{-1} d \lambda$, this implies

$$
\kappa(A)=\operatorname{rank} P_{\mathscr{N}, \mathscr{R}}=\operatorname{rank} P_{\mathscr{N}^{\prime}, \mathscr{R}^{\prime}}=\operatorname{dim} \mathscr{N}_{m}=\operatorname{dim} \mathscr{N}_{m}^{\prime}
$$

Remark that if $A_{0}$ is Fredholm of index $0, \operatorname{Im} A_{0}$ is closed and $\alpha_{0}^{\perp}=\left(\operatorname{Im} A_{0}\right)^{\perp}$, $\beta_{0}^{\perp}=\operatorname{Ker} A_{0}$ are finite dimensional; hence Assumption 2.1 is satisfied.

A direct consequence of Theorems 2.5 and 2.6 is the following.

Corollary 6.1 (Rank of $Z_{n}, n=0, \ldots, m-1$, and dimension of $\mathscr{N}_{m}, \mathscr{N}_{m}^{\prime}$ ). Assume that $A_{0}$ is Fredholm of index 0 and let $r_{h}=\operatorname{dim} \alpha_{h}=\operatorname{dim} \beta_{h}, h=1, \ldots, m$. Then

$$
\begin{array}{cc}
0 \leq r_{h}<\infty, \quad h=1, \ldots, m-1, & 0<r_{m}<\infty, \\
\operatorname{rank} Z_{n}=\sum_{h=0}^{n}(h+1) r_{m-n+h}<\infty, & n=0, \ldots, m-1,
\end{array}
$$

and

$$
\operatorname{dim} \mathscr{N}_{m}=\operatorname{dim} \mathscr{N}_{m}^{\prime}=\sum_{h=1}^{m} h r_{h}<\infty
$$

This expresses the rank of the operators in the principal part of the resolvent and the dimension of the subspaces in (1.6) in terms of the quantities defined by the local subspace decomposition.

Combining (6.1) and Corollary 6.1, one can express $\kappa(A)$ as follows.

Corollary 6.2 (Algebraic multiplicity of $A(\lambda)$ at 0 ). If $A_{0}$ is Fredholm of index 0 , then the algebraic multiplicity of $A(\lambda)$ at 0 is equal to $\sum_{h=1}^{m} h r_{h}<\infty$.

Finally it is shown that the local subspace decomposition fully describes the partial multiplicities; this is a direct consequence of Theorem 2.4, which states that in the Gohberg and Sigal [17] factorization one can choose $D(\lambda)=\sum_{h=0}^{m} P_{\beta_{h}} \lambda^{h}$ or $D(\lambda)=$ $\sum_{h=0}^{m} P_{\alpha_{h}} \lambda^{h}$.

Corollary 6.3 (Partial multiplicities of $A(\lambda)$ at 0 ). If $A_{0}$ is Fredholm of index 0 , then the number of distinct partial multiplicities of $A(\lambda)$ at 0 is equal to $\sum_{h=1}^{m} 1_{r_{h}>0}$ and there are exactly $r_{h}<\infty$ partial multiplicities that are equal to $h=1, \ldots, m$.

Remark that $r_{m}>0$ but $r_{h}$ can be 0 for some $h=1, \ldots, m-1$; in this case, no partial multiplicities is equal to $h$. The results in Corollaries 6.1, 6.2 and 6.3 are collected together in Theorem 2.7. 


\section{Declaration of competing interest}

There is no competing interest.

\section{Appendix A. Proofs}

Proof of Lemma 4.1. The proof of (4.1) is by induction and consists in showing that the $Y$ version of equation $n=0, \ldots, m$ in system (1.8) implies

$$
S_{h} Z_{n-h}+P_{a_{h}^{\perp}} Q_{h+1} Z_{n-h-1}=1_{n=m} P_{a_{h}^{\perp}}, \quad h=0,1, \ldots, n, \quad n=0, \ldots, m
$$

replacing $n$ with $n+h$ one finds (4.1). In order to show that (A.1) holds for $h=0$, observe that the $Y$ version of equation $n$ in system (1.8) reads $A_{0} Z_{n}+A_{1} Z_{n-1}=1_{n=m} I$. By definition, $P_{a_{0}^{\perp}}=I, S_{0}=A_{0}$ and $Q_{1}=A_{1}$ and this shows that (A.1) holds for $h=0$. Next assume that (A.1) holds for $h=0, \ldots, \ell-1$ and show that it also holds for $h=\ell \leq n$. First note that $S_{h}^{+} S_{h}=P_{\beta_{h}}$ and $S_{h}^{+} P_{a_{h}^{\perp}}=S_{h}^{+}$; hence applying $S_{h}^{+}$on both sides of (A.1), by the induction assumption one has that the $Y$ version of equation $n=0, \ldots, m$ in system (1.8) implies

$$
P_{\beta_{h}} Z_{n-h}+S_{h}^{+} Q_{h+1} Z_{n-h-1}=1_{n=m} S_{h}^{+}, \quad h=0,1, \ldots, \ell-1, \quad n=0, \ldots, m .
$$

Observe that, because $n-\ell+h \leq n-1<m$ for $h=0,1, \ldots, \ell-1$, in equation $n-\ell+h$ one has $1_{n-\ell+h=m}=0$ and hence

$$
P_{\beta_{h}} Z_{n-\ell}=-S_{h}^{+} Q_{h+1} Z_{n-\ell-1}, \quad h=0,1, \ldots, \ell-1 .
$$

Next write (A.1) for $h=\ell-1$,

$$
S_{\ell-1} Z_{n-\ell+1}+P_{a_{\ell-1}^{\perp}} Q_{\ell} Z_{n-\ell}=1_{n=m} P_{a_{\ell-1}^{\perp}},
$$

where $\operatorname{Im} S_{\ell-1}=\alpha_{\ell-1}$, see Definition 2.2; applying $P_{a_{\ell}^{\perp}}$, where $a_{\ell}=\bigoplus_{h=0}^{\ell-1} \alpha_{h}$, on both sides of this equation and using $P_{a_{\ell}^{\perp}} S_{\ell-1}=0$ and $P_{a_{\ell}^{\perp}} P_{a_{\ell-1}^{\perp}}=P_{a_{\ell}^{\perp}}$ one has

$$
P_{a_{\ell}^{\perp}} Q_{\ell} Z_{n-\ell}=1_{n=m} P_{a_{\ell}^{\perp}}
$$

Next consider $b_{\ell}=\bigoplus_{h=0}^{\ell-1} \beta_{h}$ and use projections, inserting $I=P_{b_{\ell}^{\perp}}+P_{b_{\ell}}$ between $Q_{\ell}$ and $Z_{n-\ell}$; one finds

$$
P_{a_{\ell}^{\perp}} Q_{\ell} Z_{n-\ell}=\left(P_{a_{\ell}^{\perp}} Q_{\ell} P_{b_{\ell}^{\perp}}\right) Z_{n-\ell}+P_{a_{\ell}^{\perp}} Q_{\ell} P_{b_{\ell}} Z_{n-\ell}
$$

Because $P_{b_{\ell}}=\sum_{j=0}^{\ell-1} P_{\beta_{j}}$, one has $P_{a_{\ell}{ }^{\perp}} Q_{\ell} P_{b_{\ell}} Z_{n-\ell}=P_{a_{\ell}^{\perp}} Q_{\ell} \sum_{j=0}^{\ell-1} P_{\beta_{j}} Z_{n-\ell}$ and by the induction assumption, see (A.2), one finds 


$$
P_{a_{\ell} \perp} Q_{\ell} P_{b_{\ell}} Z_{n-\ell}=-P_{a_{\ell}^{\perp}}\left(Q_{\ell} \sum_{j=0}^{\ell-1} S_{j}^{+} Q_{j+1}\right) Z_{n-\ell-1} .
$$

By Definition 2.2, one has $P_{a_{\ell}^{\perp}} Q_{\ell} P_{b_{\ell}^{\perp}}=S_{\ell}$ and $Q_{\ell+1}=-Q_{\ell} \sum_{j=0}^{\ell-1} S_{j}^{+} Q_{j+1}$ and hence one rewrites (A.3) as

$$
S_{\ell} Z_{n-\ell}+P_{a_{\ell}^{\perp}} Q_{\ell+1} Z_{n-\ell-1}=1_{n=m} P_{a_{\ell}^{\perp}}
$$

This shows that (A.1) holds for $h=\ell$ and completes the proof of (4.1). The proof of (4.2) applies similar arguments to the $X$ version of system (1.8) and it is omitted.

The proof of Theorem 2.3 makes use of the following lemma.

Lemma A.1. Consider Definition 2.2. Then $\operatorname{Im} Z_{0} \subseteq b_{m}^{\perp}$ and $a_{m} \subseteq \operatorname{Ker} Z_{0}$.

Proof. For $n=0,(4.1)$ and (4.2) read

$$
S_{h} Z_{0}=1_{h=m} P_{a \frac{\perp}{h}}, \quad Z_{0} S_{h}=1_{h=m} P_{b_{h}^{\perp}} \quad h=0, \ldots, m
$$

and imply

$$
S_{h} Z_{0}=Z_{0} S_{h}=0, \quad h=0, \ldots, m-1 .
$$

From $S_{h} Z_{0}=0, h=0, \ldots, m-1$, one has $\operatorname{Im} Z_{0} \subseteq\left(\bigcap_{h=0}^{m-1} \operatorname{Ker} S_{h}\right)$. By Definition 2.2, Ker $S_{h}=\beta_{h}^{\perp}$ and hence $\operatorname{Im} Z_{0} \subseteq \bigcap_{h=0}^{m-1} \beta_{h}^{\perp}=\left(\bigoplus_{h=0}^{m-1} \beta_{h}\right)^{\perp}=b_{m}^{\perp}$. This proves the first statement. From $Z_{0} S_{h}=0, h=0, \ldots, m-1$, one has $\bigoplus_{h=0}^{m-1} \operatorname{Im} S_{h} \subseteq \operatorname{Ker} Z_{0}$. By Definition 2.2, $\operatorname{Im} S_{h}=\alpha_{h}$ and hence $\bigoplus_{h=0}^{m-1} \alpha_{h}=a_{m} \subseteq \operatorname{Ker} Z_{0}$. This completes the proof.

Proof of Theorem 2.3. First note that by definition, $(i)$ holds if and only if the identity is in equation $m$ of system (1.8).

$(i) \Leftrightarrow(i i)$ : It is shown that $(i) \Rightarrow(i i) \Rightarrow\left(\operatorname{Im} Z_{0}=\beta_{m}\right) \Rightarrow(i)$. Under $(i)$, one has $h=m$ in (A.4), i.e. $Z_{0} S_{m}=P_{b_{m}^{\perp}}, b_{m}^{\perp} \neq 0$; by Definition 2.2, $b_{m} \subseteq \operatorname{Ker} S_{m}$ and because $b_{m} \subset$ Ker $S_{m}$ contradicts $Z_{0} S_{m}=P_{b_{m}^{\perp}}$, one has $b_{m}=\operatorname{Ker} S_{m}$. By Definition 2.2, Ker $S_{m}=\beta_{m}^{\perp}$ and $b_{m}=\beta_{0} \oplus \cdots \oplus \beta_{m-1}$, and hence $(i i)$. Moreover, by Lemma A.1, $\operatorname{Im} Z_{0} \subseteq b_{m}^{\perp}$ and because $\operatorname{Im} Z_{0} \subset b_{m}^{\perp}$ contradicts $Z_{0} S_{m}=P_{b_{m}^{\perp}}$, one has $\operatorname{Im} Z_{0}=b_{m}^{\perp}$. Using $b_{m}^{\perp}=\beta_{m}$, see (ii), one finds $\operatorname{Im} Z_{0}=\beta_{m}$. Next let $\operatorname{Im} Z_{0}=\beta_{m}$ and proceed by contradiction, assuming that the identity is not in equation $m$, so that the first equation in (A.4) reads $S_{m} Z_{0}=0$, which implies $\operatorname{Im} Z_{0} \subseteq \operatorname{Ker} S_{m}$, where $\operatorname{Im} Z_{0}=\beta_{m}$ and $\operatorname{Ker} S_{m}=\beta_{m}^{\perp}$. Hence $\beta_{m} \subseteq \beta_{m}^{\perp}$, so that $\beta_{m}=0$. This contradicts $Z_{0} \neq 0$, i.e. that the pole has order $m$, and proves that (i) holds. 
$(i) \Leftrightarrow\left(\right.$ iii): It is shown that $(i) \Rightarrow($ iii $) \Rightarrow\left(\operatorname{Ker} Z_{0}=\alpha_{m}^{\perp}\right) \Rightarrow(i)$. Under $(i)$, one has $h=m$ in (A.4), i.e. $S_{m} Z_{0}=P_{a_{m}^{\perp}}, a_{m}^{\perp} \neq 0$; by Definition 2.2, $\operatorname{Im} S_{m} \subseteq a_{m}^{\perp}$ and because $\operatorname{Im} S_{m} \subset a_{m}^{\perp}$ contradicts $S_{m} Z_{0}=P_{a_{m}^{\perp}}$, one has $\operatorname{Im} S_{m}=a_{m}^{\perp}$. By Definition 2.2, $\operatorname{Im} S_{m}=\alpha_{m}$ and $a_{m}^{\perp}=\left(\alpha_{0} \oplus \cdots \oplus \alpha_{m-1}\right)^{\perp}$, and hence (iii). Moreover, by Lemma A.1, $a_{m} \subseteq \operatorname{Ker} Z_{0}$ and because $a_{m} \subset \operatorname{Ker} Z_{0}$ contradicts $S_{m} Z_{0}=P_{a_{m}^{\perp}}$, one has $a_{m}=\operatorname{Ker} Z_{0}$. Using $a_{m}=\alpha_{m}^{\perp}$, see $(i i i)$, one finds $\operatorname{Ker} Z_{0}=\alpha_{m}^{\perp}$. Next let $\operatorname{Ker} Z_{0}=\alpha_{m}^{\perp}$ and proceed by contradiction, assuming that the identity is not in equation $m$, so that the second equation in (A.4) reads $Z_{0} S_{m}=0$, which implies $\operatorname{Im} S_{m} \subseteq \operatorname{Ker} Z_{0}$, where $\operatorname{Im} S_{m}=\alpha_{m}$ and Ker $Z_{0}=\alpha_{m}^{\perp}$. Hence $\alpha_{m} \subseteq \alpha_{m}^{\perp}$, so that $\alpha_{m}=0$ and thus $\alpha_{m}^{\perp}=Y$. This contradicts $Z_{0} \neq 0$, i.e. that the pole has order $m$, and proves that $(i)$ holds.

Finally note that $S_{m} Z_{0}=P_{\alpha_{m}}$ implies $Z_{0} S_{m} Z_{0}=Z_{0} P_{\alpha_{m}}$ and because $Z_{0}=Z_{0} P_{\alpha_{m}}$ one finds $Z_{0} S_{m} Z_{0}=Z_{0}$; similarly, from $Z_{0} S_{m}=P_{\beta_{m}}$ one has $S_{m} Z_{0} S_{m}=S_{m} P_{\beta_{m}}$ and because $S_{m}=S_{m} P_{\beta_{m}}$ one finds $S_{m} Z_{0} S_{m}=S_{m}$. Hence $Z_{0}$ is the generalized inverse of $S_{m}$.

Proof of Proposition 4.2. It is first shown that $F_{h}(\lambda) A(\lambda)^{-1}=\lambda^{-h} \widetilde{F}_{h}(\lambda), h=0, \ldots, m$. Applying $P_{\beta_{h}}$ on both sides of (1.5) one obtains

$$
P_{\beta_{h}} A(\lambda)^{-1}=\sum_{n=0}^{\infty} P_{\beta_{h}} Z_{n} \lambda^{-m+n} .
$$

First consider $h=m$; setting $n=0$ and $h=m$ in (4.3) one finds $P_{\beta_{m}} Z_{0}=S_{m}^{+}$. This shows that (A.5) implies $F_{m}(\lambda) A(\lambda)^{-1}=\lambda^{-m} \widetilde{F}_{m}(\lambda)$, where $F_{m}(\lambda)=P_{\beta_{m}}$ and $\widetilde{F}_{m}(0)=S_{m}^{+}$.

Next consider $h=m-1$; setting $n=0$ and $h=m-1$ in (4.3) one finds $P_{\beta_{m-1}} Z_{0}=0$ and setting $n=1$ and $h=m-1$ in (4.3) one has $P_{\beta_{m-1}} Z_{1}=S_{m-1}^{+}-S_{m-1}^{+} Q_{m} Z_{0}$. This shows that (A.5) implies

$$
P_{\beta_{m-1}} A(\lambda)^{-1}=\left(S_{m-1}^{+}-S_{m-1}^{+} Q_{m} Z_{0}\right) \lambda^{-m+1}+\sum_{n=2}^{\infty} P_{\beta_{m-1}} Z_{n} \lambda^{-m+n} .
$$

Applying $\lambda S_{m-1}^{+} Q_{m}$ on both sides of $A(\lambda)^{-1}=\sum_{n=0}^{\infty} Z_{n} \lambda^{-m+n}$ one obtains

$$
\left(\lambda S_{m-1}^{+} Q_{m}\right) A(\lambda)^{-1}=S_{m-1}^{+} Q_{m} Z_{0} \lambda^{-m+1}+\sum_{n=1}^{\infty} S_{m-1}^{+} Q_{m} Z_{n} \lambda^{-m+n+1}
$$

and summing these two expressions one has

$$
\left(P_{\beta_{m-1}}+\lambda S_{m-1}^{+} Q_{m}\right) A(\lambda)^{-1}=S_{m-1}^{+} \lambda^{-m+1}+\sum_{n=2}^{\infty}\left(P_{\beta_{m-1}} Z_{n}+S_{m-1}^{+} Q_{m} Z_{n-1}\right) \lambda^{-m+n} .
$$

This shows that (A.5) implies $F_{m-1}(\lambda) A(\lambda)^{-1}=\lambda^{-m+1} \widetilde{F}_{m-1}(\lambda)$, where $F_{m-1}(\lambda)=$ $P_{\beta_{m-1}}+\lambda S_{m-1}^{+} Q_{m}$ and $\widetilde{F}_{m-1}(0)=S_{m-1}^{+}$. 
Finally consider $h=0, \ldots, m-2$. Write $A(\lambda)^{-1}=\sum_{n=0}^{\infty} Z_{n} \lambda^{-m+n}$ as

$$
A(\lambda)^{-1}=Z_{0} \lambda^{-m}+\sum_{n=1}^{m-h-1} Z_{n} \lambda^{-m+n}+\lambda^{-h} R_{0}(\lambda), \quad R_{0}(0)=Z_{h}
$$

and apply $P_{\beta_{h}}$ on both sides to find

$$
P_{\beta_{h}} A(\lambda)^{-1}=P_{\beta_{h}} Z_{0} \lambda^{-m}+\sum_{n=1}^{m-h-1} P_{\beta_{h}} Z_{n} \lambda^{-m+n}+\lambda^{-h} P_{\beta_{h}} R_{0}(\lambda)
$$

Setting $n=0$ in (4.3) one has $P_{\beta_{h}} Z_{0}=0$ and hence

$$
P_{\beta_{h}} A(\lambda)^{-1}=\sum_{n=1}^{m-h-1} P_{\beta_{h}} Z_{n} \lambda^{-m+n}+\lambda^{-h} P_{\beta_{h}} R_{0}(\lambda)
$$

Because $1_{n+h=m}=0$ for $n=1, \ldots, m-h-1$, from $(4.3)$ one has $P_{\beta_{h}} Z_{n}=-S_{h}^{+} Q_{h+1} Z_{n-1}$ and hence

$$
\sum_{n=1}^{m-h-1} P_{\beta_{h}} Z_{n} \lambda^{-m+n}=-S_{h}^{+} Q_{h+1}\left(\sum_{n=1}^{m-h-1} Z_{n-1} \lambda^{-m+n}\right) .
$$

Next write

$$
\lambda A(\lambda)^{-1}=\left(\sum_{n=1}^{m-h-1} Z_{n-1} \lambda^{-m+n}\right)+\lambda^{-h} R_{1}(\lambda), \quad R_{1}(0)=Z_{h-1},
$$

so that

$$
\sum_{n=1}^{m-h-1} P_{\beta_{h}} Z_{n} \lambda^{-m+n}=-\lambda S_{h}^{+} Q_{h+1} A(\lambda)^{-1}+\lambda^{-h} S_{h}^{+} Q_{h+1} R_{1}(\lambda) .
$$

Substituting this expression in (A.6) and rearranging one finds $F_{h}(\lambda) A(\lambda)^{-1}=$ $\lambda^{-h} \widetilde{F}_{h}(\lambda)$, where

$$
F_{h}(\lambda)=P_{\beta_{h}}+\lambda S_{h}^{+} Q_{h+1}, \quad \widetilde{F}_{h}(\lambda)=P_{\beta_{h}} R_{0}(\lambda)+S_{h}^{+} Q_{h+1} R_{1}(\lambda) .
$$

Note that, because $R_{0}(0)=Z_{h}$ and $R_{1}(0)=Z_{h-1}$, one has

$$
\widetilde{F}_{h}(0)=P_{\beta_{h}} Z_{h}+S_{h}^{+} Q_{h+1} Z_{h-1}=S_{h}^{+},
$$

where the last equality follows setting $n=m-h$ in (4.3). This shows that

$$
F_{h}(\lambda) A(\lambda)^{-1}=\lambda^{-h} \widetilde{F}_{h}(\lambda), \quad h=0, \ldots, m,
$$


where $F_{h}(\lambda)=P_{\beta_{h}}+1_{h \neq m} S_{h}^{+} Q_{h+1} \lambda$ and $\widetilde{F}_{h}(0)=S_{h}^{+}$, i.e. $F_{h}(\lambda) \in \mathscr{B}_{X}$ is a root function of order $-h$ of $A(\lambda)^{-1}$ at 0 . This completes the proof of the second equality in (4.6). The proof of the first equality in (4.6) applies similar arguments to $A(\lambda)^{-1} P_{\alpha_{h}}=$ $\sum_{n=0}^{\infty} Z_{n} P_{\alpha_{h}} \lambda^{-m+n}$ using (4.4) and it is omitted.

Proof of Theorem 2.4. It is first shown that $A(\lambda)=E_{\beta}(\lambda) D_{\beta}(\lambda) F_{\beta}(\lambda)$. From (4.6) one has

$$
\widetilde{F}_{h}(\lambda) A(\lambda)=\lambda^{h} F_{h}(\lambda), \quad \widetilde{F}_{h}(0)=S_{h}^{+}, \quad h=0, \ldots, m,
$$

and summing over $h$ one finds

$$
\widetilde{F}(\lambda) A(\lambda)=\sum_{h=0}^{m} \lambda^{h} F_{h}(\lambda), \quad \widetilde{F}(\lambda)=\sum_{h=0}^{m} \widetilde{F}_{h}(\lambda) .
$$

First it is shown that $\widetilde{F}(0)=\sum_{h=0}^{m} \widetilde{F}_{h}(0)=\sum_{h=0}^{m} S_{h}^{+}$is invertible: because $\operatorname{Im} S_{h}^{+}=\beta_{h}$ and $X=\bigoplus_{h=0}^{m} \beta_{h}$, see $(i i)$ in Theorem 2.3, $\widetilde{F}(0)$ is onto. Because Ker $S_{h}^{+}=\alpha_{h}^{\perp}$ and $Y=\bigoplus_{h=0}^{m} \alpha_{h}$, see (iii) in Theorem 2.3, one has

$$
0=\left(\bigoplus_{h=0}^{m} \alpha_{h}\right)^{\perp}=\bigcap_{h=0}^{m} \alpha_{h}^{\perp}=\bigcap_{h=0}^{m} \operatorname{Ker} S_{h}^{+}=\operatorname{Ker} \widetilde{F}(0)
$$

i.e. $\widetilde{F}(0)$ is one-to-one. This shows that $\widetilde{F}(0)$ is invertible and thus $\widetilde{F}(\lambda)$ is analytic and invertible on $D_{\rho}$. Note that

$$
\begin{aligned}
& \left(\sum_{h=0}^{m} S_{h}\right)\left(\sum_{h=0}^{m} S_{h}^{+}\right)=\sum_{h=0}^{m} S_{h} S_{h}^{+}=\sum_{h=0}^{m} P_{\alpha_{h}}=I, \\
& \left(\sum_{h=0}^{m} S_{h}^{+}\right)\left(\sum_{h=0}^{m} S_{h}\right)=\sum_{h=0}^{m} S_{h}^{+} S_{h}=\sum_{h=0}^{m} P_{\beta_{h}}=I,
\end{aligned}
$$

i.e.

$$
\left(\sum_{h=0}^{m} S_{h}\right)^{-1}=\sum_{h=0}^{m} S_{h}^{+}
$$

Next it is shown that $\widetilde{F}(\lambda) A(\lambda)=D_{\beta}(\lambda) F_{\beta}(\lambda)$. Consider the r.h.s. of (A.7); using the definition of $F_{h}(\lambda)$ in (4.5), this can be written as

$$
\sum_{h=0}^{m} \lambda^{h} F_{h}(\lambda)=P_{\beta_{0}}+\sum_{h=1}^{m}\left(P_{\beta_{h}}+S_{h-1}^{+} Q_{h}\right) \lambda^{h} .
$$


On the other hand, the definitions of $D_{\beta}(\lambda)$ and $F_{\beta}(\lambda)$ imply

$$
D_{\beta}(\lambda) F_{\beta}(\lambda)=P_{\beta_{0}}+\sum_{h=1}^{m}\left(P_{\beta_{h}}-P_{\beta_{h-1}} M_{\beta}\right) \lambda^{h}-P_{\beta_{m}} M_{\beta} \lambda^{m+1},
$$

where $P_{\beta_{h-1}} M_{\beta}=-P_{\beta_{h-1}} \sum_{j=0}^{m-1} S_{j}^{+} Q_{j+1}=-P_{\beta_{h-1}} S_{h-1}^{+} Q_{h}=-S_{h-1}^{+} Q_{h}$ for $h=$ $1, \ldots, m$ and $P_{\beta_{m}} M_{\beta}=-P_{\beta_{m}} \sum_{j=0}^{m-1} S_{j}^{+} Q_{j+1}=0$ because $\operatorname{Im} S_{h}^{+}=\beta_{h}$. Hence

$$
D_{\beta}(\lambda) F_{\beta}(\lambda)=P_{\beta_{0}}+\sum_{h=1}^{m}\left(P_{\beta_{h}}+S_{h-1}^{+} Q_{h}\right) \lambda^{h}=\sum_{h=0}^{m} \lambda^{h} F_{h}(\lambda) .
$$

This shows that $\widetilde{F}(\lambda) A(\lambda)=D_{\beta}(\lambda) F_{\beta}(\lambda)$, i.e. $A(\lambda)=E_{\beta}(\lambda) D_{\beta}(\lambda) F_{\beta}(\lambda)$, where

$$
E_{\beta}(\lambda)=\widetilde{F}(\lambda)^{-1}, \quad D_{\beta}(\lambda)=\sum_{h=0}^{m} P_{\beta_{h}} \lambda^{h}, \quad F_{\beta}(\lambda)=I-M_{\beta} \lambda .
$$

Note that $E_{\beta}(\lambda)$ and $F_{\beta}(\lambda)$ are analytic and invertible on $D_{\rho}$ because $F_{\beta}(0)=I$ and $E_{\beta}(0)=\widetilde{F}(0)^{-1}=\left(\sum_{h=0}^{m} S_{h}^{+}\right)^{-1}=\sum_{h=0}^{m} S_{h}$. This completes the proof of $(2.2)$ for $\zeta=\beta$. The proof of $A(\lambda)=E_{\alpha}(\lambda) D_{\alpha}(\lambda) F_{\alpha}(\lambda)$ applies similar arguments to $A(\lambda) \widetilde{E}_{h}(\lambda)=$ $\lambda^{h} E_{h}(\lambda), \widetilde{E}_{h}(0)=S_{h}^{+}, h=0, \ldots, m$, and it is omitted.

Next it is shown that the operators $E_{\beta, n}$ in $E_{\beta}(\lambda)=\sum_{n=0}^{\infty} E_{\beta, n} \lambda^{n}$ satisfy the formulas given in the statement. From $E_{\beta}(0)=\sum_{h=0}^{m} S_{h}$ one has $E_{\beta, 0}=\sum_{h=0}^{m} S_{h}$ and because $E_{\beta}(\lambda)=A(\lambda) F_{\beta}(\lambda)^{-1} D_{\beta}(\lambda)^{-1}=\sum_{n=0}^{\infty} E_{\beta, n} \lambda^{n}$, the formula for $E_{\beta, n}, n=1,2, \ldots$, is found as follows. Let $B_{\beta}(\lambda)=F_{\beta}(\lambda)^{-1} D_{\beta}(\lambda)^{-1}$; from $F_{\beta}(\lambda)^{-1}=\sum_{n=0}^{\infty} M_{\beta}^{n} \lambda^{n}$ and $D_{\beta}(\lambda)^{-1}=\sum_{h=0}^{m} P_{\beta_{h}} \lambda^{-h}$ one has

$$
B_{\beta}(\lambda)=F_{\beta}(\lambda)^{-1} D_{\beta}(\lambda)^{-1}=\left(\sum_{n=0}^{\infty} M_{\beta}^{n} \lambda^{n}\right)\left(\sum_{h=0}^{m} P_{\beta_{h}} \lambda^{-h}\right)=\sum_{n=0}^{\infty} B_{\beta, n} \lambda^{n-m},
$$

where

$$
B_{\beta, n}=\left\{\begin{array}{cl}
\sum_{k=0}^{n} M_{\beta}^{k} P_{\beta_{m-n+k}}, & n=0, \ldots, m \\
M_{\beta}^{n-m} B_{\beta, m}, & n=m+1, m+2, \ldots
\end{array} .\right.
$$

Hence

$$
E_{\beta}(\lambda)=A(\lambda) F_{\beta}(\lambda)^{-1} D_{\beta}(\lambda)^{-1}=\left(A_{0}+A_{1} \lambda\right) \sum_{n=0}^{\infty} B_{\beta, n} \lambda^{n-m}
$$

implies

$$
\begin{array}{cc}
A_{0} B_{\beta, n}+A_{1} B_{\beta, n-1}=0, & n=0, \ldots, m, \\
E_{\beta, n}= & A_{0} B_{\beta, m+n}+A_{1} B_{\beta, m+n-1}, \quad n=0,1, \ldots,
\end{array}
$$


having defined $B_{\beta,-1}=0$, i.e.

$$
E_{\beta, n}=\left(A_{0} M_{\beta}+A_{1}\right) M_{\beta}^{n-1} B_{\beta, m}, \quad n=1,2, \ldots
$$

Because $M_{\beta}=-\sum_{h=0}^{m-1} S_{h}^{+} Q_{h+1}$, see Definition 2.2, Ker $A_{0}=\beta_{1} \oplus \cdots \oplus \beta_{m}$ and $\operatorname{Im} S_{h}^{+}=\beta_{h}$, one has $A_{0} M_{\beta}=-A_{0} S_{0}^{+} Q_{1}$. Recall that $S_{0}=A_{0}$ and $Q_{1}=A_{1}$, see Definition 2.2, so that $A_{0} S_{0}^{+} Q_{1}=P_{\alpha_{0}} A_{1}$; hence $A_{0} M_{\beta}+A_{1}=\left(I-P_{\alpha_{0}}\right) A_{1}=P_{\alpha_{0}^{\perp}} A_{1}$. Thus

$$
E_{\beta, n}=P_{\alpha_{0}^{\perp}} A_{1} M_{\beta}^{n-1} B_{\beta, m}, \quad n=1,2, \ldots
$$

This completes the proof for $E_{\beta, n}, n=0,1, \ldots$ The derivation of the formulas for $F_{\alpha, n}$ in the statement applies similar arguments to $F_{\alpha}(\lambda)=D_{\alpha}(\lambda)^{-1} E_{\alpha}(\lambda)^{-1} A(\lambda)=$ $\sum_{n=0}^{\infty} F_{\alpha, n} \lambda^{n}$ and it is omitted. It introduces the notation

$$
C_{\alpha}(\lambda)=D_{\alpha}(\lambda)^{-1} E_{\alpha}(\lambda)^{-1}=\sum_{n=0}^{\infty} C_{\alpha, n} \lambda^{n-m}
$$

where

$$
C_{\alpha, n}=\left\{\begin{array}{cl}
\sum_{k=0}^{n} P_{\alpha_{m-n+k}}\left(M_{\alpha}^{*}\right)^{k}, & n=0, \ldots, m \\
C_{\alpha, m}\left(M_{\alpha}^{*}\right)^{n-m}, & n=m+1, m+2, \ldots
\end{array} .\right.
$$

Proof of Corollary 4.3. From (2.2) one has

$$
A(\lambda)^{-1}=B_{\beta}(\lambda) C_{\beta}(\lambda)=B_{\alpha}(\lambda) C_{\alpha}(\lambda)=\sum_{n=0}^{\infty} Z_{n} \lambda^{n-m}
$$

where

$$
\begin{aligned}
& B_{\beta}(\lambda)=F_{\beta}(\lambda)^{-1} D_{\beta}(\lambda)^{-1}=\sum_{n=0}^{\infty} B_{\beta, n} \lambda^{n-m}, \\
& C_{\beta}(\lambda)=E_{\beta}(\lambda)^{-1}=\sum_{n=0}^{\infty} C_{\beta, n} \lambda^{n}, \\
& B_{\alpha}(\lambda)=F_{\alpha}(\lambda)^{-1}=\sum_{n=0}^{\infty} B_{\alpha, n} \lambda^{n} \\
& C_{\alpha}(\lambda)=D_{\alpha}(\lambda)^{-1} E_{\alpha}(\lambda)^{-1}=\sum_{n=0}^{\infty} C_{\alpha, n} \lambda^{n-m} .
\end{aligned}
$$


The expressions of $B_{\beta, n}$ and $C_{\alpha, n}$ are given in (A.9) and in (A.12) and those of $E_{\beta, k}$ and $F_{\alpha, k}$ are taken from Theorem 2.4. Because $E_{\beta}(0)=F_{\alpha}(0)=\sum_{h=0}^{m} S_{h}$ is invertible, those of $C_{\beta, n}$ and $B_{\alpha, n}$ are found from $E_{\beta}(\lambda) C_{\beta}(\lambda)=F_{\alpha}(\lambda) B_{\alpha}(\lambda)=I$, i.e. $\sum_{k=0}^{n} E_{\beta, k} C_{\beta, n-k}=\sum_{k=0}^{n} F_{\alpha, k} B_{\alpha, n-k}=1_{n=0} I$.

Proof of Proposition 5.1. We first prove the statement for the $X$-chains. From (2.2) one has $A(\lambda) F_{\beta}(\lambda)^{-1}=E_{\beta}(\lambda) D_{\beta}(\lambda)$, and substituting $F_{\beta}(\lambda)^{-1}=\sum_{n=0}^{\infty} M_{\beta}^{n} \lambda^{n}$ and $D_{\beta}(\lambda)=$ $\sum_{h=0}^{m} P_{\beta_{h}} \lambda^{h}$ one finds $A(\lambda)\left(\sum_{n=0}^{\infty} M_{\beta}^{n} \lambda^{n}\right)=E_{\beta}(\lambda)\left(\sum_{h=0}^{m} P_{\beta_{h}} \lambda^{h}\right)$, which implies

$$
A(\lambda)\left(\sum_{n=0}^{\infty} M_{\beta}^{n} P_{\beta_{h}} \lambda^{n}\right)=\lambda^{h} E_{\beta}(\lambda) P_{\beta_{h}}
$$

where $E_{\beta}(0) P_{\beta_{h}}=S_{h}$. Substituting $A(\lambda)=A_{0}+\lambda A_{1}$ and rearranging, one rewrites the l.h.s. of (A.13) as

$$
\left(A_{0}+\lambda A_{1}\right)\left(\sum_{n=0}^{\infty} M_{\beta}^{n} P_{\beta_{h}} \lambda^{n}\right)=A_{0} P_{\beta_{h}}+\sum_{n=1}^{\infty}\left(A_{0} M_{\beta}^{n}+A_{1} M_{\beta}^{n-1}\right) P_{\beta_{h}} \lambda^{n}
$$

thus (A.13) implies $A_{0} P_{\beta_{h}}=0$ and

$$
\left(A_{0} M_{\beta}^{n}+A_{1} M_{\beta}^{n-1}\right) P_{\beta_{h}}=\left\{\begin{array}{ll}
0, & n=1, \ldots, h-1 \\
S_{h}, & n=h
\end{array}, \quad h=1, \ldots, m .\right.
$$

That is,

$$
A_{0} x_{h, 0}=0, \quad A_{0} x_{h, n}+A_{1} x_{h, n-1}=\left\{\begin{array}{cl}
0, & n=1, \ldots, h-1 \\
S_{h} x_{h, 0} \neq 0, & n=h
\end{array}\right.
$$

where $x_{h, 0}$ is a nonzero vector in $\beta_{h}$ and $x_{h, n}=M_{\beta}^{n} x_{h, 0}$. This completes the proof of the first part of the statement.

In order to prove the second part of the statement, we wish to show that the vectors $v_{1}, v_{2}, \ldots, v_{m}$, where $v_{h}=\sum_{n=1}^{h} c_{h, n} x_{h, n-1}$ for some scalars $c_{h, n}$, are linearly independent. Suppose not, i.e. assume that there exists a linear combination $w=\sum_{h=1}^{m} b_{h} v_{h}$ such that $w=0$ for some scalars $b_{1}, \ldots, b_{m}$ not all equal to 0 . Letting $\mathcal{H}=\left\{h=1, \ldots, m: b_{h} \neq 0\right\}$ be the set of indices of the nonzero scalars in the linear combination, one has $w=\sum_{h \in \mathcal{H}} b_{h} v_{h}, b_{h} \neq 0$.

Consider $P_{\alpha_{0}^{\perp}} A_{1} w=\sum_{h \in \mathcal{H}} b_{h} w_{h}$, where $w_{h}=P_{\alpha_{0}^{\perp}} A_{1} v_{h}$, and observe that (A.14) implies

$$
P_{\alpha_{0}^{\perp}} A_{1} x_{h, n-1}=\left\{\begin{array}{cl}
0, & n=1, \ldots, h-1 \\
S_{h} x_{h, 0} \neq 0, & n=h
\end{array},\right.
$$


because $P_{\alpha_{0}^{\perp}} A_{0}=0$ and $P_{\alpha_{0}^{\perp}} S_{h}=S_{h}$.

Hence $w_{h}=P_{\alpha_{0}^{\perp}} A_{1} v_{h}=\sum_{n=1}^{h} c_{h, n} P_{\alpha_{0}^{\perp}} A_{1} x_{h, n-1}=c_{h, h} S_{h} x_{h, 0}$, see (A.15). From $w=$ 0 it follows that $0=P_{\alpha_{0}^{\perp}} A_{1} w=\sum_{h \in \mathcal{H}} b_{h} w_{h}, b_{h} \neq 0$, and because the $\alpha$-subspaces are orthogonal and $w_{h} \in \alpha_{h}$, this contradicts $w=0$ unless $w_{h}=0$. Since $w_{h}=c_{h, h} S_{h} x_{h, 0}=$ 0 if and only if $c_{h, h}=0$, it must be that $v_{h}=\sum_{n=1}^{h-1} c_{h, n} x_{h, n-1}$.

Then consider $P_{\alpha_{0}^{\perp}} A_{1} M_{\beta} w=\sum_{h \in \mathcal{H}} b_{h} u_{h}$, where $u_{h}=P_{\alpha_{0}^{\perp}} A_{1} M_{\beta} v_{h}$; because $M_{\beta} v_{h}=\sum_{n=1}^{h-1} c_{h, n} M_{\beta} x_{h, n-1}=\sum_{n=1}^{h-1} c_{h, n} x_{h, n}$, one has $u_{h}=P_{\alpha_{0}^{\perp}} A_{1} M_{\beta} v_{h}=$ $\sum_{n=1}^{h-1} c_{h, n} P_{\alpha_{0}^{\perp}} A_{1} x_{h, n}=c_{h, h-1} S_{h} x_{h, 0}$, see (A.15). From $w=0$ it follows that $0=$ $P_{\alpha_{0}^{\perp}} A_{1} M_{\beta} w=\sum_{h \in \mathcal{H}} b_{h} u_{h}, b_{h} \neq 0$, and because the $\alpha$-subspaces are orthogonal and $u_{h} \in \alpha_{h}$, this contradicts $w=0$ unless $u_{h}=0$. Since $u_{h}=c_{h, h-1} S_{h} x_{h, 0}=0$ if and only if $c_{h, h-1}=0$, it must be that $v_{h}=\sum_{n=1}^{h-2} c_{h, n} x_{h, n}$. Iterating on the same reasoning, one finds $c_{h, h-2}=\cdots=c_{h, 1}=0$. This shows that $0=w=\sum_{h \in \mathcal{H}} b_{h} v_{h}, b_{h} \neq 0$, implies $v_{h}=0$ hence reaching a contradiction. This proves that the vectors $v_{1}, v_{2}, \ldots, v_{m}$ are linearly independent and completes the proof of the statement for the $X$-chains.

The proof of the statement on the $Y$-chains (the explicit steps are omitted) applies similar arguments to $E_{\alpha}(\lambda)^{-1} A(\lambda)=D_{\alpha}(\lambda) F_{\alpha}(\lambda)$, see (2.2), and arrives at $P_{\alpha_{h}} A_{0}=0$ and

$$
P_{\alpha_{h}}\left(\left(M_{\alpha}^{*}\right)^{n} A_{0}+\left(M_{\alpha}^{*}\right)^{n-1} A_{1}\right)=\left\{\begin{array}{ll}
0, & n=1, \ldots, h-1 \\
S_{h}, & n=h
\end{array}, \quad h=1, \ldots, m\right.
$$

and taking adjoint one has $A_{0}^{*} P_{\alpha_{h}}=0$ and

$$
\left(A_{0}^{*} M_{\alpha}^{n}+A_{1}^{*} M_{\alpha}^{n-1}\right) P_{\alpha_{h}}=\left\{\begin{array}{rl}
0, & n=1, \ldots, h-1 \\
S_{h}^{*}, & n=h
\end{array}, \quad h=1, \ldots, m .\right.
$$

Hence

$$
A_{0}^{*} y_{h, 0}=0, \quad A_{0}^{*} y_{h, n}+A_{1}^{*} y_{h, n-1}\left\{\begin{array}{cl}
0, & n=1, \ldots, h-1 \\
S_{h}^{*} y_{h, 0} \neq 0, & n=h
\end{array}\right.
$$

where $y_{h, 0}$ is a nonzero vector in $\alpha_{h}$ and $y_{h, n}=M_{\alpha}^{n} y_{h, 0}$.

Proof of Theorem 2.5. Given $n \geq 1$ and vectors $\xi_{0}, \ldots, \xi_{n}$ in $X$, Bart and Lay [10] call $\left(\xi_{0}, \ldots, \xi_{n}\right)$ is a chain of length $n+1$ if $A_{0} \xi_{j}+A_{1} \xi_{j+1}=0$ for $j=0, \ldots, n-1$. Here we let $\varphi_{n-j}=\xi_{j}, j=0, \ldots, n$, and write the chain in reverse order $\left(\varphi_{0}, \ldots, \varphi_{n}\right)=$ $\left(\xi_{n}, \ldots, \xi_{0}\right)$, so that $A_{0} \varphi_{j+1}+A_{1} \varphi_{j}=0$ for $j=0, \ldots, n-1$. In the present notation, the characterization of $\mathscr{N}_{n}$ given in Bart and Lay [10] is as follows

$$
\mathscr{N}_{n}=\left\{x \in X: \exists \text { chain }\left(\varphi_{0}, \ldots, \varphi_{n}\right) \text { such that } \varphi_{n}=x \text { and } \varphi_{0}=0\right\}
$$

That is, the chains that are used to construct $\mathscr{N}_{n}, n=1, \ldots, m$, are such that 


$$
A_{0} \varphi_{1}=0, \quad A_{0} \varphi_{j+1}+A_{1} \varphi_{j}=0, \quad j=1, \ldots, n-1, \quad n=1, \ldots, m .
$$

From Proposition 5.1, (replacing $n$ with $k$ ) one has

$$
A_{0} x_{h, 0}=0, \quad A_{0} x_{h, k}+A_{1} x_{h, k-1}=0, \quad k=1, \ldots, h-1, \quad h=1, \ldots, m,
$$

where $x_{h, 0}$ a nonzero vector in $\beta_{h}$ and $x_{h, k}=M_{\beta}^{k} x_{h, 0}$, and

$$
A_{0} x_{h, h}+A_{1} x_{h, h-1} \neq 0, \quad h=1, \ldots, m .
$$

Hence $\varphi_{1}$ can be chosen equal to $x_{h, 0}$ for $h=1, \ldots, m$, i.e. $\mathscr{N}_{1}=\bigoplus_{h=1}^{m} \beta_{h}, \varphi_{2}$ can be chosen equal to $x_{h, 1}=M_{\beta} x_{h, 0}$ for $h=2, \ldots, m$ and to $x_{h, 0}$ for $h=1, \ldots, m$ (setting $\left.\varphi_{1}=0\right)$, i.e. $\mathscr{N}_{2}=\left(\bigoplus_{h=2}^{m} M_{\beta} \beta_{h}\right) \oplus \mathscr{N}_{1}=\bigoplus_{h=1}^{m} \bigoplus_{k=0}^{\min (h-1,1)} M_{\beta} \beta_{h}, \varphi_{3}$ can be chosen equal to $x_{h, 2}=M_{\beta}^{2} x_{h, 0}$ for $h=3, \ldots, m$, to $x_{h, 1}=M_{\beta} x_{h, 0}$ for $h=2, \ldots, m$ (setting $\varphi_{2}=0$ ) and to $x_{h, 0}$ for $h=1, \ldots, m$ (setting $\left.\varphi_{1}=\varphi_{2}=0\right)$, i.e. $\mathscr{N}_{3}=\left(\bigoplus_{h=3}^{m} M_{\beta}^{2} \beta_{h}\right) \oplus$ $\mathscr{N}_{2}=\bigoplus_{h=1}^{m} \bigoplus_{k=0}^{\min (h-1,2)} M_{\beta}^{k} \beta_{h}$, and so on. This shows that

$$
\mathscr{N}_{n}=\bigoplus_{h=1}^{m} \bigoplus_{k=0}^{\min (h-1, n-1)} M_{\beta}^{k} \beta_{h}, \quad n=1, \ldots, m
$$

Hence

$$
\mathscr{N}_{m}=\bigoplus_{h=1}^{m} \bigoplus_{k=0}^{h-1} M_{\beta}^{k} \beta_{h}, \quad \mathscr{N}_{m}^{\prime}=A_{1} \mathscr{N}_{m}=A_{1} \bigoplus_{h=1}^{m} \bigoplus_{k=0}^{h-1} M_{\beta}^{k} \beta_{h}
$$

Applying a similar reasoning to $A(\lambda)^{*}=A_{0}^{*}+A_{1}^{*} \lambda$ with decompositions $Y=\widetilde{\mathscr{N}}_{m} \oplus \widetilde{\mathscr{R}}_{m}$, $X=\widetilde{\mathscr{N}_{m}^{\prime}} \oplus \widetilde{\mathscr{R}}_{m}^{\prime}$, where $\widetilde{\mathscr{N}}, \widetilde{\mathscr{R}}$ correspond to $\mathscr{N}, \mathscr{R}$ in the Bart and Lay [10] construction, one finds

$$
\widetilde{\mathscr{N}_{n}}=\bigoplus_{h=1}^{m} \bigoplus_{k=0}^{\min (h-1, n-1)} M_{\alpha}^{k} \alpha_{h}, \quad n=1, \ldots, m
$$

and hence

$$
\widetilde{\mathscr{N}_{m}}=\bigoplus_{h=1}^{m} \bigoplus_{k=0}^{h-1} M_{\alpha}^{k} \alpha_{h}, \quad \widetilde{\mathscr{N}_{m}^{\prime}}=A_{1}^{*} \widetilde{\mathscr{N}_{m}}=A_{1}^{*} \bigoplus_{h=1}^{m} \bigoplus_{k=0}^{h-1} M_{\alpha}^{k} \alpha_{h}
$$

Because $Y=\widetilde{\mathscr{N}_{m}} \oplus \widetilde{\mathscr{R}}_{m}=\mathscr{N}_{m}^{\prime} \oplus \mathscr{R}_{m}^{\prime}$ and $X=\widetilde{\mathscr{N}}_{m}^{\prime} \oplus \widetilde{\mathscr{R}}_{m}^{\prime}=\mathscr{N}_{m} \oplus \mathscr{R}_{m}$, where $\widetilde{\mathscr{N}_{m}}\left(\widetilde{\mathscr{R}}_{m}\right)$ is isomorphic to $\mathscr{N}_{m}^{\prime}\left(\mathscr{R}_{m}^{\prime}\right)$ and $\widetilde{\mathscr{N}}_{m}^{\prime}\left(\widetilde{\mathscr{R}}_{m}^{\prime}\right)$ is isomorphic to $\mathscr{N}_{m}\left(\mathscr{R}_{m}\right)$, one can take

$$
\mathscr{R}_{m}^{\prime}=\left(\widetilde{\mathscr{N}_{m}}\right)^{\perp}=\left(\bigoplus_{h=1}^{m} \bigoplus_{k=0}^{h-1} M_{\alpha}^{k} \alpha_{h}\right)^{\perp}, \quad \mathscr{R}_{m}=\left(\widetilde{\mathscr{N}_{m}^{\prime}}\right)^{\perp}=\left(A_{1}^{*} \bigoplus_{h=1}^{m} \bigoplus_{k=0}^{h-1} M_{\alpha}^{k} \alpha_{h}\right)^{\perp}
$$


Proof of Theorem 2.6. From Corollary 4.3 one has

$$
Z_{n}=\sum_{k=0}^{n} B_{\beta, k} C_{\beta, n-k}=\sum_{k=0}^{n} B_{\alpha, n-k} C_{\alpha, k}, \quad n=0,1, \ldots
$$

where

$$
\begin{gathered}
B_{\beta, k}=\left\{\begin{array}{c}
\sum_{j=0}^{k} M_{\beta}^{j} P_{\beta_{m-k+j}} \\
M_{\beta}^{k-m} B_{\beta, m}
\end{array}\right. \\
C_{\alpha, k}=\left\{\begin{array}{cc}
\sum_{j=0}^{k} P_{\alpha_{m-k+j}}\left(M_{\alpha}^{*}\right)^{j} & k=0, \ldots, m \\
C_{\alpha, m}\left(M_{\alpha}^{*}\right)^{k-m} & k=m+1, m+2, \ldots .
\end{array}\right.
\end{gathered}
$$

Observe that, because of linear independence of the $X$ and $Y$-chains, one has

$$
\operatorname{Im} Z_{n}=\bigoplus_{k=0}^{n} \operatorname{Im} B_{\beta, k}, \quad \operatorname{Im} Z_{n}^{*}=\bigoplus_{k=0}^{n} \operatorname{Im} C_{\alpha, k}^{*}, \quad n=0, \ldots, m-1
$$

where

$$
\operatorname{Im} B_{\beta, k}=\bigoplus_{j=0}^{k} M_{\beta}^{j} \beta_{m-k+j}, \quad \operatorname{Im} C_{\alpha, k}^{*}=\bigoplus_{j=0}^{k} M_{\alpha}^{j} \alpha_{m-k+j}, \quad k=0, \ldots, m-1
$$

Thus

$$
\operatorname{Im} Z_{n}=\bigoplus_{h=m-n}^{m} \bigoplus_{k=0}^{h-m+n} M_{\beta}^{k} \beta_{h}, \quad \operatorname{Im} Z_{n}^{*}=\bigoplus_{h=m-n}^{m} \bigoplus_{k=0}^{h-m+n} M_{\alpha}^{k} \alpha_{h}, \quad n=0, \ldots, m-1
$$

Because $\operatorname{Ker} Z_{n}=\left(\operatorname{Im} Z_{n}^{*}\right)^{\perp}$, this completes the proof of the statement.

Proof of Corollary 5.2. From $\operatorname{Im} Z_{n}=\bigoplus_{k=0}^{n} \operatorname{Im} B_{\beta, k}, n=0, \ldots, m-1$, see (A.16), one has

$$
\operatorname{Im} Z_{n}=\left(\bigoplus_{k=0}^{n-1} \operatorname{Im} B_{\beta, k}\right) \oplus \operatorname{Im} B_{\beta, n}=\operatorname{Im} Z_{n-1} \oplus \xi_{\beta, n}, \quad n=1, \ldots, m-1
$$

where $\xi_{\beta, n}=\operatorname{Im} B_{\beta, n} \supseteq M_{\beta}^{n} \beta_{m} \neq 0$ because of linear independence of the $X$-chains. This proves the statement about $\operatorname{Im} Z_{n}$. Similarly, from the second equation in (A.16) one has

$$
\operatorname{Im} Z_{n}^{*}=\operatorname{Im} Z_{n-1}^{*} \oplus \xi_{\alpha, n}, \quad n=1, \ldots, m-1,
$$

where $\xi_{\alpha, n}=\operatorname{Im} C_{\alpha, n}^{*} \supseteq M_{\alpha}^{n} \alpha_{m} \neq 0$ because of linear independence of the $Y$-chains. Hence 


$$
\left(\operatorname{Im} Z_{n}^{*}\right)^{\perp}=\left(\operatorname{Im} Z_{n-1}^{*} \oplus \xi_{\alpha, n}\right)^{\perp}=\left(\operatorname{Im} Z_{n-1}^{*}\right)^{\perp} \cap \xi_{\alpha, n}^{\perp},
$$

i.e.

$$
\operatorname{Ker} Z_{n}=\operatorname{ker} Z_{n-1} \cap \xi_{\alpha, n}^{\perp}, \quad n=1, \ldots, m-1 .
$$

Proof of Corollary 6.1. Because of linear independence of the vectors in the $X$-chains, one has $\operatorname{dim}\left(M_{\beta}^{k} \beta_{h}\right)=\operatorname{dim} \beta_{h}=r_{h}$ and

$$
\operatorname{dim} \operatorname{Im} Z_{n}=\sum_{h=m-n}^{m} \sum_{k=0}^{h-m+n} \operatorname{dim}\left(M_{\beta}^{k} \beta_{h}\right)=\sum_{h=m-n}^{m}(h-m+n+1) r_{h}
$$

i.e. $\operatorname{rank} Z_{n}=\sum_{h=0}^{n}(h+1) r_{m-n+h}, n=0, \ldots, m-1$. Because $\operatorname{dim} \mathscr{N}_{m}=\operatorname{dim} \mathscr{N}_{m}^{\prime}=$ rank $Z_{m-1}$, this proves the statement.

Proof of Corollary 6.3. Let $q$ be the number of distinct partial multiplicities of $A(\lambda)$ at 0 and for $h=1, \ldots, q$, let $m_{h}$ be one of the distinct partial multiplicities. That is, write $0<\kappa_{1} \leq \kappa_{2} \leq \cdots \leq \kappa_{s}<\infty$ as $0<m_{1}<m_{2}<\cdots<m_{q}<\infty$, where $m_{1}=\kappa_{1}$ and $m_{q}=\kappa_{s}$ and let $s_{h}$ be the number of non distinct partial multiplicities $\kappa_{j}$ that are equal to $m_{h}$. Consider $D(\lambda)=D_{0}+D_{1} \lambda^{\kappa_{1}}+\cdots+D_{s} \lambda^{\kappa_{s}}$ and sum the projections that load the same partial multiplicity into $D(\lambda)$, i.e. let

$$
\begin{gathered}
M_{0}=D_{0}, \quad M_{1}=D_{1}+\cdots+D_{s_{1}} \\
M_{2}=D_{s_{1}+1}+\cdots+D_{s_{1}+s_{2}}, \quad \ldots \quad
\end{gathered}
$$

so that $D(\lambda)=M_{0}+M_{1} \lambda^{m_{1}}+\cdots+M_{q} \lambda^{m_{q}}$, where $M_{0}, M_{1}, \ldots, M_{q}$ are mutually disjoint projections that decompose the identity, $M_{0}$ is Fredholm of index 0, and rank $M_{h}=s_{h}<$ $\infty, h=1, \ldots, q$. Remark that $q,\left\{m_{1}, m_{2}, \cdots, m_{q}\right\}$ and $\left\{s_{1}, s_{2}, \cdots, s_{q}\right\}$ are uniquely defined. By Theorem 2.4 one can take $D(\lambda)=\sum_{h=0}^{m} P_{\beta_{h}} \lambda^{h}\left(\right.$ or $\left.D(\lambda)=\sum_{h=0}^{m} P_{\alpha_{h}} \lambda^{h}\right)$, where $\operatorname{dim} \alpha_{h}=\operatorname{dim} \beta_{h}=r_{h}$; hence one has that $q=\sum_{h=1}^{m} 1_{\left\{r_{h}>0\right\}},\left\{m_{1}, m_{2}, \cdots, m_{q}\right\}=$ $\left\{h=1, \ldots, m: r_{h}>0\right\}$ and $\left\{s_{1}, s_{2}, \cdots, s_{q}\right\}=\left\{r_{h}, h=1, \ldots, m: r_{h}>0\right\}$. Thus the statement.

Proof of Theorem 2.7. See the proofs of Corollaries 6.1, 6.2 and 6.3.

\section{References}

[1] A. Albrecht, P. Howlett, C. Pearce, Necessary and sufficient conditions for the inversion of linearlyperturbed bounded linear operators on Banach space using Laurent series, J. Math. Anal. Appl. 383 (2011) 95-110.

[2] A. Albrecht, P. Howlett, C. Pearce, The fundamental equations for inversion of operator pencils on Banach space, J. Math. Anal. Appl. 413 (2014) 411-421.

[3] A. Albrecht, P. Howlett, G. Verma, The fundamental equations for the generalized resolvent of an elementary pencil in a unital Banach algebra, Linear Algebra Appl. 574 (2019) 216-251. 
[4] A. Albrecht, P. Howlett, G. Verma, Inversion of operator pencils on Banach space using Jordan chains when the generalized resolvent has an isolated essential singularity, Linear Algebra Appl. 595 (2020) 33-62.

[5] K. Avrachenkov, J. Filar, P. Howlett, Analytic Perturbation Theory and Its Applications, Society for Industrial and Applied Mathematics, Philadelphia, PA, 2013.

[6] K. Avrachenkov, M. Haviv, P. Howlett, Inversion of analytic matrix functions that are singular at the origin, SIAM J. Matrix Anal. Appl. 22 (2001) 1175-1189.

[7] H. Bart, T. Ehrhardt, B. Silbermann, Zero sums of idempotents and Banach algebras failing to be spectrally regular, in: Advances in Structured Operator Theory and Related Areas, in: Operator Theory: Advances and Applications, vol. 237, Birkhäuser, Basel, 2013, pp. 41-78.

[8] H. Bart, T. Ehrhardt, B. Silbermann, Sums of idempotents and logarithmic residues in zero pattern matrix algebras, Linear Algebra Appl. 498 (2016) 262-316.

[9] H. Bart, T. Ehrhardt, B. Silbermann, How small can a sum of idempotents be?, Integral Equ. Oper. Theory 92 (2020) 10.

[10] H. Bart, D. Lay, Poles of a generalised resolvent operator, Proc. R. Ir. Acad., A Math. Phys. Sci. 74 (1974) 147-168.

[11] M. Franchi, Some results on eigenvalues of finite type, resolvents and Riesz projections, Linear Algebra Appl. 588 (2020) 238-271.

[12] M. Franchi, P. Paruolo, Inversion of regular analytic matrix functions: local Smith form and subspace duality, Linear Algebra Appl. 435 (2011) 2896-2912.

[13] M. Franchi, P. Paruolo, Inverting a matrix function around a singularity via local rank factorization, SIAM J. Matrix Anal. Appl. 37 (2016) 774-797.

[14] M. Franchi, P. Paruolo, Cointegration in functional autoregressive processes, Econom. Theory 36 (2020) 803-839.

[15] I. Gohberg, S. Goldberg, M. Kaashoek, Basic Classes of Linear Operators, Birkhäuser, Basel-BostonBerlin, 2003.

[16] I. Gohberg, M. Krein, The basic propositions on defect numbers, root numbers and indices of linear operators, Usp. Mat. Nauk 12 (1957) 43-118 (Russian) [English transl.:] AMS Translations, Ser. 2, vol. 13, pp. 185-264 (1960).

[17] I. Gohberg, E. Sigal, On operator generalizations of the logarithmic residue theorem and the theorem of Rouché, Math. USSR Sb. 13 (4) (1971) 603-625.

[18] S. Goldberg, Unbounded Linear Operators: Theory and Applications, McGraw-Hill, New York, 1966.

[19] M. Haviv, Y. Ritov, On series expansions and stochastic matrices, SIAM J. Matrix Anal. Appl. 14 (1993) 670-676.

[20] M. Haviv, Y. Ritov, U. Rothblum, Taylor expansions of eigenvalues of perturbed matrices with applications to spectral radii of nonnegative matrices, Linear Algebra Appl. 168 (1992) 159-188.

[21] P. Howlett, Input retrieval in finite dimensional linear systems, J. Aust. Math. Soc. Ser. B, Appl. Math. 23 (1982) 357-382.

[22] P. Howlett, K. Avrachenkov, C. Pearce, V. Ejov, Inversion of analytically perturbed linear operators that are singular at the origin, J. Math. Anal. Appl. 353 (2009) 68-84.

[23] M. Kaashoek, Ascent, descent, nullity and defect, a note on a paper by A.E. Taylor, Math. Ann. 172 (1967) 105-115.

[24] T. Kato, Perturbation theory for nullity, deficiency and other quantities of linear operators, J. Anal. Math. 6 (1958) 261-322.

[25] C. Langenhop, The Laurent expansion for a nearly singular matrix, Linear Algebra Appl. 4 (1971) $329-340$.

[26] C. Langenhop, On the invertibility of a nearly singular matrix, Linear Algebra Appl. 7 (1973) $361-365$.

[27] D. Lay, Spectral analysis using ascent, descent, nullity and defect, Math. Ann. 184 (1970) $197-214$.

[28] P. Schweitzer, G. Stewart, The Laurent expansion of pencils that are singular at the origin, Linear Algebra Appl. 183 (1993) 237-254.

[29] A. Taylor, Theorems on ascent, descent, nullity and defect of linear operators, Math. Ann. 163 (1966) 18-49. 